\title{
Genotoxic stress inhibits Ewing sarcoma cell growth by modulating alternative pre-mRNA processing of the RNA helicase DHX9
}

\author{
Marco Fidaleo ${ }^{1,2, *}$, Francesca Svetoni ${ }^{1,2, *}$, Elisabetta Volpe ${ }^{2}$, Belén Miñana ${ }^{3,4}$, \\ Daniela Caporossi ${ }^{1}$, Maria Paola Paronetto ${ }^{1,2}$ \\ ${ }^{1}$ Department of Movement, Human and Health Sciences, University of Rome "Foro Italico", Rome, Italy \\ ${ }^{2}$ Laboratories of Cellular and Molecular Neurobiology and of Neuroimmunology, CERC, Fondazione Santa Lucia, Rome, Italy \\ ${ }^{3}$ Centre de Regulació Genòmica, Barcelona, Spain \\ ${ }^{4}$ Universitat Pompeu Fabra, Barcelona, Spain \\ "These authors have contributed equally to this work
}

Correspondence to:

Maria Paola Paronetto, e-mail: mariapaola.paronetto@uniroma4.it

Keywords: Ewing sarcoma, alternative splicing, DNA damage, DHX9

Received: April 13, $2015 \quad$ Accepted: September 21, 2015

Published: October 02, 2015

\section{ABSTRACT}

Alternative splicing plays a key role in the DNA damage response and in cancer. Ewing Sarcomas (ES) are aggressive tumors caused by different chromosomal translocations that yield in-frame fusion proteins driving transformation. RNA profiling reveals genes differentially regulated by UV light irradiation in two ES cell lines exhibiting different sensitivity to genotoxic stress. In particular, irradiation induces a new isoform of the RNA helicase $D H X 9$ in the more sensitive SK-N-MC cells, which is targeted to nonsense-mediated decay (NMD), causing its downregulation. DHX9 protein forms a complex with RNA polymerase II (RNAPII) and EWS-FLI1 to enhance transcription. Silencing of $D H X 9$ in ES cells sensitizes them to UV treatment and impairs recruitment of EWS-FLI1 to target genes, whereas DHX9 overexpression protects ES cells from genotoxic stress. Mechanistically, we found that UV light irradiation leads to enhanced phosphorylation and decreased processivity of RNAPII in SK-N-MC cells, which in turn causes inclusion of $D H X 9$ exon $6 \mathrm{~A}$. A similar effect on $D H X 9$ splicing was also elicited by treatment with the chemotherapeutic drug etoposide, indicating a more general mechanism of regulation in response to DNA damage. Our data identify a new NMD-linked splicing event in $D H X 9$ with impact on EWS-FLI1 oncogenic activity and ES cell viability.

\section{INTRODUCTION}

Ewing Sarcomas (ES) are aggressive tumors of bone and soft tissues. They are caused by different chromosomal translocations that yield in-frame fusion proteins comprising the amino terminus of the EWS protein fused to the carboxyl terminus of various ETS transcription factors [1]. These chimeric proteins activate a specific oncogenic program to direct neoplastic transformation [2, 3]. Among them, EWS-FLI1, generated by chromosomal translocation between chromosome 22 and 11 [3], is the most common product, representing a landmark of ES.
Both EWS and EWS-FLI1 modulate gene expression $[4,5]$. Besides transcription, recent evidence unveiled a role for both proteins in the modulation of alternative splicing (AS) of target genes [6,9]. EWS modulates AS of genes involved in the DNA damage response, including key regulators of genotoxic stress like CHEK2, ABL1 and MDM2 [8,9]. Accordingly, EWS deficiency enhances sensitivity to ionizing radiation (IR) [10] and UV light irradiation [8]. Furthermore, two highthroughput screens identified the gene encoding EWS (EWSR 1) as required for resistance to ionizing radiation (IR) [11] and camptothecin [12]. Importantly, in ES cells the intact EWSR1 gene is present only on one allele, while 
the other allele is affected by the translocation. Thus, EWSR1 haploinsufficiency might contribute, at least in part, to ES cells sensitivity to genotoxic stress.

DNA damage triggers the activation of signaling cascades that profoundly influence chromatin structure, thus modulating gene expression. Genotoxic stress imposed by irradiation or chemotherapeutic agents modulates AS events [7, 13], in part through reduced transcription elongation rates as a consequence of RNA Polymerase II (RNAPII) phosphorylation [14]. In this regard, mounting evidence points to aberrant AS regulation as a key step in oncogenesis [15] and indicates that splicing regulation represents a suitable target for therapeutic intervention [16].

Despite the reported links between EWS and the DNA damage response [7, 8, 10-12], whether or not changes in gene expression in response to genotoxic stress can affect the sensitivity of ES cells to irradiation has not been extensively investigated yet. In this work we identified changes in the transcriptome that are induced by low UV light irradiation in two ES cell lines (SK-N-MC and LAP-35 cells) displaying different sensitivity to UV light treatment. Among other targets, we found that UV light irradiation induced down-regulation of $D H X 9$ in SK-N-MC cells, partially through the generation of a new isoform that is targeted to non-mediated decay (NMD). DHX9 enhances EWS-FLI1-mediated transcription and favours anchorage-independent growth in ES cells [17]. We found that knockdown of DHX9 in ES cells rendered them more susceptible to UV treatment, whereas its overexpression protected ES cells from irradiation. Thus, our results strongly suggest a role for DHX9 as a transcriptional co-activator of EWS-FLI1 involved in the resistance to genotoxic stress of ES cells.

\section{RESULTS}

\section{SK-N-MC and LAP-35 Ewing Sarcoma cells display different resistance to UV light irradiation}

To ascertain the efficacy of UV irradiation in suppressing the growth of ES cells, we used two ES cell lines characterized by similar chromosomal translocation [t(11;22)(q24;q12)] generating the oncogenic fusion protein EWS/FLI-1 type 1 and 2 (Figure S1A). LAP-35 [18] and SK-N-MC [19] cells were exposed to either 10 or $40 \mathrm{~J} / \mathrm{m}^{2} \mathrm{UV}$ light and clonogenic survival assays were performed by monitoring colony formation 12 days after irradiation. In the absence of irradiation, SK-N-MC cells formed 3- to 4-fold more clones than LAP-35 cells (Figure 1A, 1B), although SK-N-MC colonies displayed smaller size. When cells were exposed to $10 \mathrm{~J} / \mathrm{m}^{2} \mathrm{UV}$ light irradiation, SK-N-MC cells formed only few clones, while LAP-35 cells were still able to proliferate, albeit displaying a 8-fold reduction in clone formation with respect to untreated cells (Figure 1A, 1B). Upon treatment with $40 \mathrm{~J} / \mathrm{m}^{2}$, survival of both cell lines was dramatically compromised (Figure 1A, 1B).

To corroborate the results of the colony formation assay, we performed cell proliferation assays at different time points after UV light treatment with $10 \mathrm{~J} / \mathrm{m}^{2}$. SK-N-MC cell proliferation was strongly reduced by UV treatment, while the effect on LAP-35 was milder (Figure 1C). Moreover, propidium iodide (PI) staining confirmed that viability was affected by UV light irradiation more dramatically in SK-N-MC cells than in LAP-35 cells (Figure 1D).

These results indicate that SK-N-MC cells are more sensitive than LAP-35 to UV light irradiation.

\section{Gene expression changes induced by UV light irradiation in ES cells}

To investigate whether different changes in gene expression (GE) could account for the different sensitivity of ES cells to UV light, we performed high-throughput analyses. RNA obtained from three biological replicas of SK-N-MC and LAP-35 cells treated with low doses of UV light $\left(10 \mathrm{~J} / \mathrm{m}^{2}\right)$ was hybridized to splicing sensitive microarrays featuring 1804 AS events in 482 genes encoding proteins with functions related to RNA processing and cancer $[8,14,20]$. Hybridization analysis revealed that 102 genes in SK-N-MC cells and 46 genes in LAP35 cells change their expression levels upon UV light treatment (fold change $>|1,3| ; p$ value $<0,01$; Figure 1E, Supplementary Table S1). Of these, 41 in SK-N-MC and 14 genes in LAP-35 cells displayed a fold change $>$ |2| (Figure S1B). Most genes were downregulated after treatment, with a more pronounced effect in SK-N-MC than in LAP-35 cells and with 32 genes common in the two cell lines (Figure 1E, S1C). Nevertheless, 4 genes in SK-N-MC cells (CD82, TRIB3, YWHAH, AFF2) and 3 genes in LAP-35 cells (CDKN1A, FOS, GADD45A) were upregulated (Figure S1D).

Array analysis indicated that UV irradiation exerted a more pronounced effect on AS than transcription. 437 AS events in 216 genes were affected in SK-N-MC cells, and $108 \mathrm{AS}$ events in 66 genes were affected in LAP-35 cells considering fold change $>|1,4|, z$ score $>3$; $p$ value $<0,01$ (Figure 1F; Supplementary Table S1), or 211 in SK-N-MC and 37 in LAP-35 cells considering fold change $>|2|$ (Figure S1F). UV light irradiation impacted all types of AS events present in the array (Figure S1E). Among these, 44 events in 41 genes were conserved in the two cell lines, indicating a certain degree of overlap in the splicing response of ES cells to UV irradiation. The proportion of genes that display regulation at both AS and expression levels is substantially higher in LAP-35 (37.9\%) than in the SK-N-MC cells (22.2\%) (Figure 1G, 1H). 
A

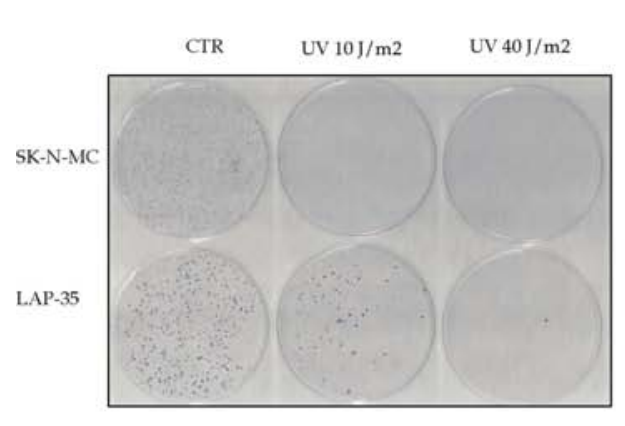

C

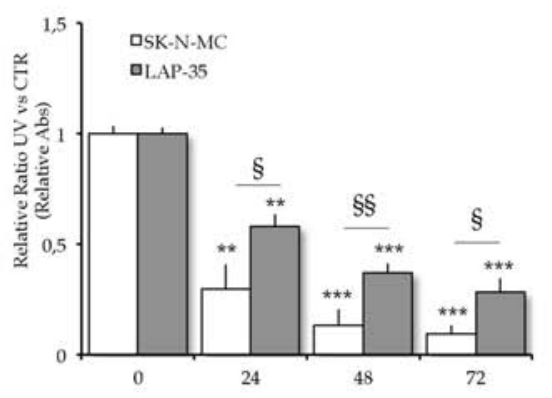

E

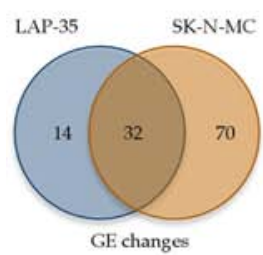

F

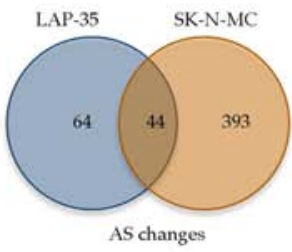

I

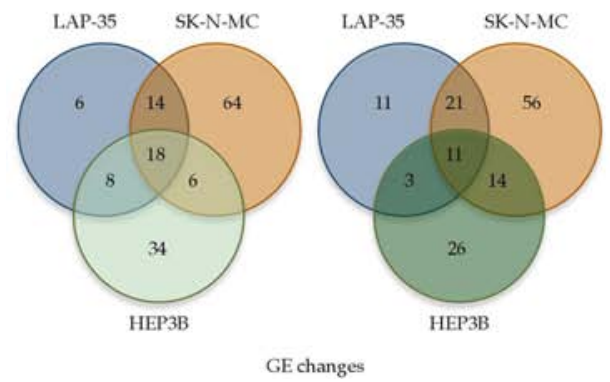

B

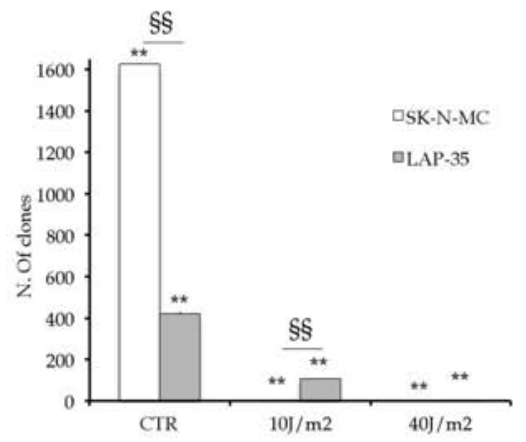

D

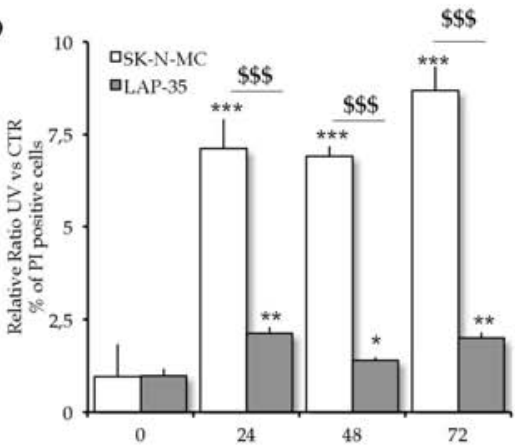

G

H

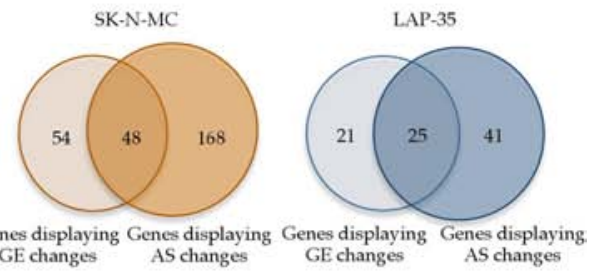

K

L

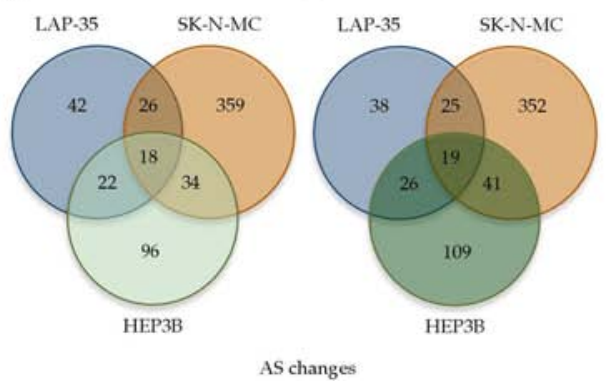

Figure 1: UV light irradiation triggers cytotoxic effect in Ewing Sarcoma cells. A. Representative images of clonogenic assays of SK-N-MC and LAP-35 cells upon UV light irradiation. B. Histograms represent colony numbers $(\mathrm{n}=3$; mean \pm s.d.) carried out on SK-N-MC (white bars) and LAP-35 cells (gray). C. Cell survival rates detected by MTS cell proliferation assay after $10 \mathrm{~J} / \mathrm{m}^{2} \mathrm{UV}$ light treatment in SK-N-MC (white) and LAP-35 cells (gray). D. Propidium Iodide (PI) viability assay; the decrease in viability was expressed as relative percentage of dead cells in treated versus control cells after $10 \mathrm{~J} / \mathrm{m}^{2} \mathrm{UV}$ light treatment in SK-N-MC (white) and LAP-35 cells (gray). In all panels statistical analysis was performed by Student's $t$-test: ${ }^{*} p<0.05,{ }^{* *} p<0.01,{ }^{* * *} p<0.001$ for CTR vs UV treatment; $\$ p<0.05, \$ \$ p<0.01, \$ \$ \$ p<0.001$ for SK-N-MC vs LAP-35 cells. E. Venn diagram shows the overlap of gene expression signatures at gene level induced by $10 \mathrm{~J} / \mathrm{m}^{2} \mathrm{UV}$ light irradiation in SK-N-MC and LAP-35 cells, as indicated. F. Venn diagram shows the overlap of gene expression signatures at AS level induced by $10 \mathrm{~J} / \mathrm{m}^{2} \mathrm{UV}$ light irradiation in SK-N-MC and LAP-35 cells, as indicated. G. and H. Venn diagrams represent the overlap of gene affected both at gene expression and AS level upon UV light irradiation in the SK-N-MC (G) and in the LAP-35 (H) cells. I. to L. Venn diagram shows the overlap of gene expression signatures at gene and AS levels induced by $10 \mathrm{~J} / \mathrm{m}^{2}(\mathrm{I}$ and $\mathrm{K}$ respectively) and $40 \mathrm{~J} / \mathrm{m}^{2}$ ( $\mathrm{J}$ and L respectively) UV light irradiation in ES cells (SK-N-MC and LAP-35) and HEP3B cells, as indicated. 
RT-quantitative PCR (RT-qPCR), using oligonucleotides covering both exons and exon-exon junctions, of all 8 randomly selected AS changes (in the C1QBP, SIP1, U2AF35, TNFRSF10B, AURKB, BAT1, $C C N A 2$ and $C R O P$ genes) identified by microarrays were validated (Figure S2A-S2H), thus confirming the fidelity of the microarray analysis. In order to evaluate the specificity of UV light response in different cancer cell lines, we performed microarray analysis of HEP3B (hepatocellular carcinoma) cells irradiated with $10 \mathrm{~J} / \mathrm{m}^{2} \mathrm{UV}$ light (Figure 1I, 1K) and also compared our datasets with a previous dataset obtained from treatment of HEP3B with $40 \mathrm{~J} / \mathrm{m}^{2} \mathrm{UV}$ light (14; Figure 1J, 1L). As expected, a more consistent overlap at GE level was observed between cells treated with the same UV intensity (Figure 1I and 1J). Interestingly, 18 genes affected at the expression level were conserved in the three cell lines (Figure 1I), and 10 of them were also affected upon $40 \mathrm{~J} / \mathrm{m}^{2}$ irradiation (not shown). Furthermore, we identified 18 AS events affected by UV light irradiation independently from the cellular context (Figure 1K), 11 of them were also conserved upon $40 \mathrm{~J} / \mathrm{m}^{2}$ irradiation (not shown). Notably, SK-N-MC cells are more responsive to UV light irradiation than other cell lines, at both GE level (102 genes affected versus 46 in LAP-35 and 66 in HEP3B) and AS level (437 AS events modulated versus 108 in LAP-35 and 170 in HEP3B; Figure 1I-1L).

\section{DHX9 is selectively regulated by UV light irradiation in SK-N-MC cells}

Among the genes that were differentially regulated in the two ES cell lines, we focused on $D H X 9$ because of its reported functional interaction with EWS-FLI1 [17]. DHX9, also known as RNA helicase A (RHA) or nuclear DNA helicase II (NDHII), displays helicase activity and modulates transcription through its interaction with RNAPII [21]. RT-qPCR analysis confirmed that UV light irradiation significantly repressed $D H X 9$ mRNA expression in SK-N-MC, but not in LAP-35 cells (Figure 2A). Notably, the microarray analysis indicated that UV light treatment also affected AS of the $D H X 9$ gene in SK-N-MC cells, leading to inclusion of exon $6 \mathrm{~A}$ and resulting in expression of a previously uncharacterized DHX9 isoform (Figure 2B). This predicted AS event was validated by RT-qPCR using different sets of primers in SK-N-MC cells, whereas it was not significantly modulated in LAP-35 cells (Figure 2B, S3A-S3E). Moreover, western blot analysis confirmed that UV treatment caused down-regulation of the DHX9 protein in SK-N-MC but not in LAP-35 cells (Figure 2C). DHX9 expression levels and exon 6A inclusion were not affected in HEP3B cell upon UV light irradiation (Figure S3G, Supplementary Table S1), suggesting that regulation of $D H X 9$ pre-mRNA processing represents a specific response of SK-N-MC ES cells to low doses of UV light.

Exon 6A contains a premature stop codon (PTC), which should lead to NMD of this new transcript (NR_033302), thus possibly contributing to the decrease in the pool of DHX9 mRNA upon UV light treatment. Indeed, UV light-induced inclusion of exon 6A in SK-N-MC cells was significantly stabilized after knockdown of UPF1, (Figure 2D-2G), an essential component of the NMD pathway [22], similarly to the effect on SRSF1 (Figure 2F), a known target of NMD [23]. These results suggest that this new DHX9 transcript is targeted by NMD.

Moreover, actinomycin D treatment at 5 hours after UV light irradiation completely abolished the accumulation of the exon 6A-containing DHX9 transcript, which returned to basal levels within 30 minutes from the block of transcription (Figure S4A). In the absence of actinomycin $\mathrm{D}$, this variant accumulates up to 10 hours after UV irradiation, and returned to basal levels by 24 hours (Figure S4A). This result indicates that expression of the NMD-targeted $D H X 9$ variant is a transient response to UV irradiation. As expected, western blot analysis indicated that knockdown of UPF1 had no effect on the UV light-induced reduction of full length DHX9 protein in SK-N-MC cells 6 hours after UV light irradiation (Figure 2H).

Collectively these results indicate that UV light irradiation causes down-regulation of DHX9 mRNA in SK-N-MC by changes in gene expression and AS-mediated NMD.

To investigate the relative stability of DHX9 protein in normal conditions and upon UV light irradiation, we treated SK-N-MC cells with cycloheximide and harvested them at different time points (2, 6 and 24 hours) after treatment. Western blot analyses revealed that full length DHX9 protein is stable in the first 6 hours of cycloheximide treatment, whereas its expression levels are halved by 24 hours of treatment (Figure S4B and S4C). However, UV light irradiation dramatically reduced the half-life of DHX9 protein, causing a 30\% reduction after 6 hours and about $80 \%$ reduction after 24 hours (Figure S4B and S4C). Notably, treatment with CHX did not further decrease the levels of DHX9, suggesting that de novo protein synthesis does not compensate the effect of UV irradiation on DHX9 protein levels (Figure S4B and S4C). Moreover, inhibition of the proteasome by treatment with MG132 prevented UV-induced DHX9 degradation and it also prevented the degradation of the caspase 3-cleaved DHX9 [24], which is induced upon UV light treatment and rapidly degraded in the absence of MG132 (Figure S4D and S4E). Collectively these experiments show that UV light irradiation induces DHX9 downregulation partly through its degradation by the proteasome and partly through induction of a new alternative isoform that is targeted by NMD, thus limiting de novo DHX9 protein synthesis.. 

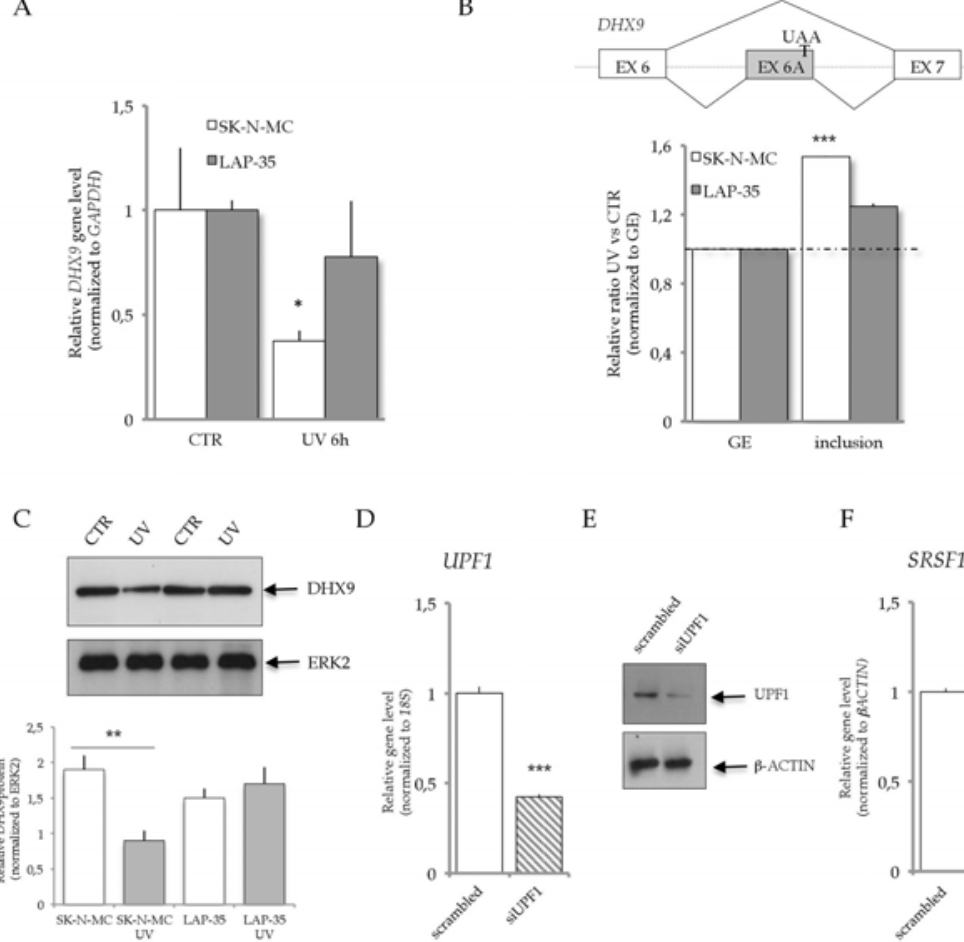

F SRSF1

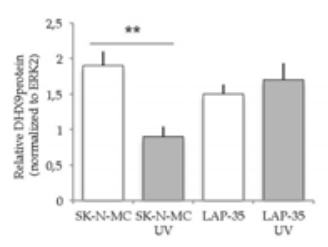

G

DHX9 (Exon 6A inclusion)

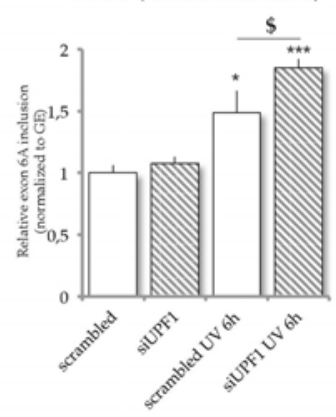

$\mathrm{H}$
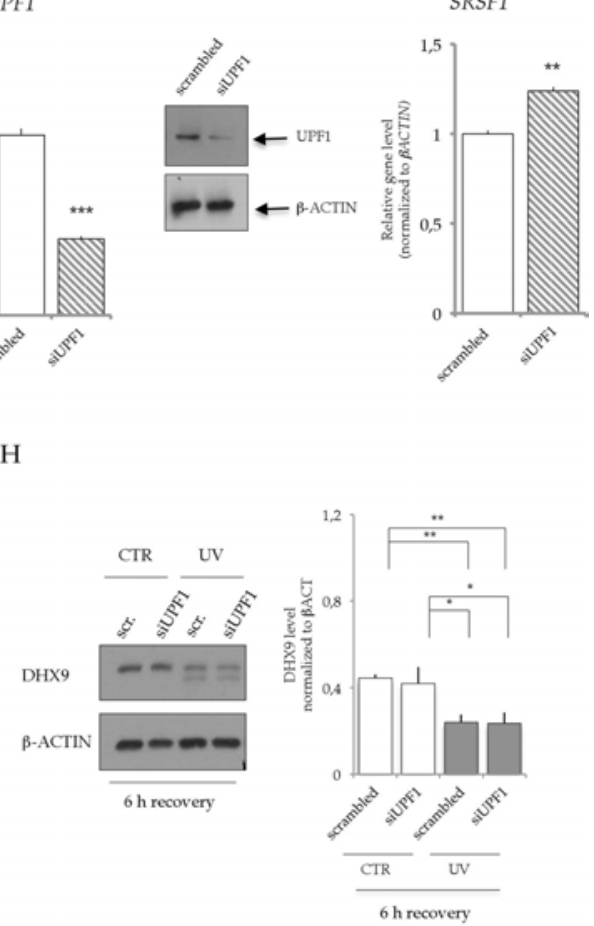

Figure 2: UV light irradiation affects $D H X 9$ mRNA expression and alternative splicing in SK-N-MC cells, but not in LAP-35. A. RT-qPCR validation of microarray-predicted GE changes in DHX9 mRNA (exon 4), normalized to GAPDH. B. Scheme of DHX9 AS event (top panel). The alternative exon 6A is upregulated in SK-N-MC cells upon UV light treatment. UAA indicates the stop codon within exon 6A. Histograms represent levels of expression of exon $6 \mathrm{~A}$ as relative ratio of cells treated with $10 \mathrm{~J} / \mathrm{m}^{2} \mathrm{UV}$ light versus untreated normalized to a constitutive exon (GE). White bars indicate SK-N-MC cells while gray bars indicate LAP-35 cells. $(n=3$; mean \pm s.d.). C. Western blot analysis of DHX9 and ERK2 expression in LAP-35 and SK-N-MC cells upon UV light irradiation. $10 \mu \mathrm{g}$ of proteins from SK-N-MC and LAP-35 cell extracts after UV light $\left(10 \mathrm{~J} / \mathrm{m}^{2}\right)$ treatment were loaded. Histograms represent the quantification of DHX9 protein normalized to ERK2. D. RT-qPCR analysis of UPF1 expression in SK-N-MC cells transfected with either scrambled (white) or siUPF1 (filled) oligonucleotides. Histograms represent UPF1 mRNA levels normalized to $18 \mathrm{~S}$ expression $(n=3$; mean $\pm \mathrm{s} . \mathrm{d})$. E. Western blot of UPF1 and $\beta$-actin expression in SK-N-MC cells transfected with either scrambled (white) or siUPF1 (filled) oligonucleotides. $10 \mu \mathrm{g}$ of proteins were loaded. F. RT-qPCR analysis to detect the expression of SRSF1, a known NMD target [23], in SK-N-MC cells transfected with either scrambled (white) or siUPF1 (filled) oligonucleotides. Histograms represent SRSF1 mRNA levels normalized to $\beta$-ACTIN expression ( $n=3$; mean \pm s.d). G. Relative DHX9 exon 6A inclusion (normalized to GE) in SK-N-MC cells, transfected either with scrambled or siUPF1 oligonucleotides, with or without UV treatment. H. Western blot analysis of DHX9 and $\beta$-ACTIN expression in SK-N-MC cells at 6 hours of recovery after UV light irradiation. $10 \mu \mathrm{g}$ of proteins from SK-N-MC cell extracts were loaded transfected either with scrambled or siUPF1 oligonucleotides. Upon UV light treatment a slower band of DHX9 protein is also induced due to caspase cleavage of the first 95 aminoacids of the protein and correlating with early stage of apoptosis, cell apoptosis as previously described [24]. On the right, histograms represent the quantification of DHX9 protein normalized to $\beta$-ACTIN from three independent experiments $(n=3$; mean \pm s.d.). In all panels statistical analysis was performed by Student's $t$-test: * $p<0.05, * * p<0.01, * * * p<0.001 ; \$ p<0.05, \$ \$ p<0.01$, $\$ \$ p<0.001$. 


\section{Reduced RNAPII processivity enhances $D H X 9$ exon 6A inclusion in SK-N-MC cells exposed to UV light irradiation}

UV light irradiation is known to widely affect gene expression, including regulation of genes involved in the DNA damage response [25]. UV irradiation induces hyper-phosphorylation of the carboxy terminal domain (CTD) of RNAPII, followed by its ubiquitylation and degradation $[14,26]$. Importantly, the phosphorylation status of the RNAPII CTD correlates with distinct functions of the polymerase, as the hypo-phosphorylated form (RNAPIIA) is associated with transcriptional initiation whereas the hyper-phosphorylated form (RNAPIIO) correlates with transcriptional elongation [27]. UV light irradiation can also influence splicing decisions through modulation of the RNAPII elongation rate [14, 28-29], representing an example of kinetic coupling between transcription and splicing. Upon genotoxic stress, phosphorylation of RNAPII CTD slows down the polymerase, offering a time window opportunity for weak exons to be efficiently recognized by the splicing machinery [14]. DHX9 exon 6A displays a low score for the 5'splice site (5.9; AAG | GTCAGT) if compared with the constitutive downstream exon (12.4; CAG | GTAAGT; see Material and Methods for calculation details), rendering it relatively weak. Thus, we asked whether the differential regulation of DHX9 AS and expression by UV light in ES cells correlated with differential effects on RNAPII phosphorylation. Western blot analysis using an antibody against total RNAPII, which recognizes both RNAPIIO and RNAPIIA, showed that RNAPII is strongly downregulated in SK-N-MC cells after 3-6 hours from UV light treatment, whereas this effect was weaker and more transient in LAP-35 cells (Figure 3A; Figure S5A, S5B). Moreover, the phosphorylation status of the CTD (RNAPIIO) was different in the two cell lines. RNAPIIO peaked at 1-3 hours after UV light treatment in SK-N-MC cells, and then it was strongly reduced (Figure 3A, 3B); on the contrary, CTD phosphorylation was only slightly increased in LAP-35 cells and it was maintained almost constant in the 24 hours following the UV light treatment (Figure 3A, 3B).

The different dynamics of RNAPII phosphorylation in the two ES cell lines exposed to UV irradiation raises the possibility that $D H X 9$ expression and exon $6 \mathrm{~A}$ splicing are affected by modulation of RNAPII elongation rate in SK-N-MC. To test this hypothesis, we first analyzed the accumulation of distal and proximal $D H X 9$ pre-mRNA and calculated RNAPII processivity as ratio between them (Figure 3C) [30]. Remarkably, UV light irradiation significantly reduced RNAPII processivity within the DHX9 transcription unit in SK-N-MC cells but not in LAP-35 cells (Figure 3C).

To further test our hypothesis, we analyzed RNAPII occupancy in the DHX9 transcription unit upon UV light irradiation by chromatin immunoprecipitation experiments (ChIP) in SK-N-MC cells. Although RNAPII binding to $D H X 9$ gene was generally decreased by UV treatment, we observed a peak in occupancy near the alternative exon $6 \mathrm{~A}$ after 6 hours of treatment (Figure 3D, left panel). Moreover, by using phospho-specific antibodies we found that UV treatment caused a progressive increase in the pausing (Ser5 phosphorylated) RNAPII, with maximal enrichment in the variant exon 6A (Figure 3D, right panel). By contrast, RNAPII phosphorylated in Ser2, which correlates with the elongating phase of the polymerase [31], was only marginally affected by the treatment (Figure $3 \mathrm{D}$, right panel). This result indicates that $\mathrm{UV}$ treatment causes a local pausing of RNAPII near exon 6A.

Next, we asked whether $D H X 9$ exon $6 \mathrm{~A}$ inclusion was favoured by the lower processivity of RNAPII induced by UV-light irradiation in SK-N-MC cells. To this end, we pharmacologically inhibited RNAPII elongation in LAP-35 cells by treatment with 5, 6-Dichlorobenzimidazole1-b-D-ribofuranoside (DRB) $[32,33]$ in the absence of genotoxic stress. RT-qPCR analysis confirmed that DRB treatment reduced RNAPII processivity in LAP-35 cells (Figure 3E). Strikingly, this was sufficient to induce exon 6A inclusion (Figure 3F) and downregulation of $D H X 9$ mRNA and protein in these cells (Figure 3G, 3H).

These experiments argue that modulation of RNAPII processivity upon UV light irradiation in SK-N-MC cells promotes inclusion of exon $6 \mathrm{~A}$ in the $\mathrm{DHX} 9$ gene, contributing to down-regulation of its expression.

\section{UV light irradiation affects EWS-FLI1 recruitment on target genes}

DHX9 protein directly interacts with EWS-FLI1 and enhances EWS-FLI1-mediated transcription, cooperating with EWS-FLI1-induced oncogenic transformation [17]. Thus, we asked if UV light irradiation affected transcription of EWS-FLI1 target genes through $D H X 9$ downregulation. Cyclin D1 (CCND1), c-MYC, and DNA-binding protein inhibitor ID-2 (ID2) genes encode proteins strictly involved in cell proliferation and cancer development [34]. In particular, ID2 expression is upregulated in ES cells as a result of both direct EWS-FLI1 binding to its promoter and increased $c-M Y C$ expression [34]. RT-qPCR analysis revealed a strong downregulation of ID2 expression $(60 \%$ relative to untreated) upon UV irradiation in SK-N-MC cells, whereas the gene was unaffected in LAP-35 cells. Furthermore, $c-M Y C$ and $C C N D 1$ genes were downregulated at higher extent in SK-N-MC (72\% and $71 \%$, respectively) than in LAP-35 cells (38\% and $32 \%$, respectively; Figure 4A). To corroborate our results we analyzed five more genes known to be direct target of EWSFLI1. Notably, the expression of EZH2, NKX2-2, NROB1, and $S O X 2$ was specifically decreased in SK-N-MC cells by UV light treatment, whereas $P D G F C$ expression was 


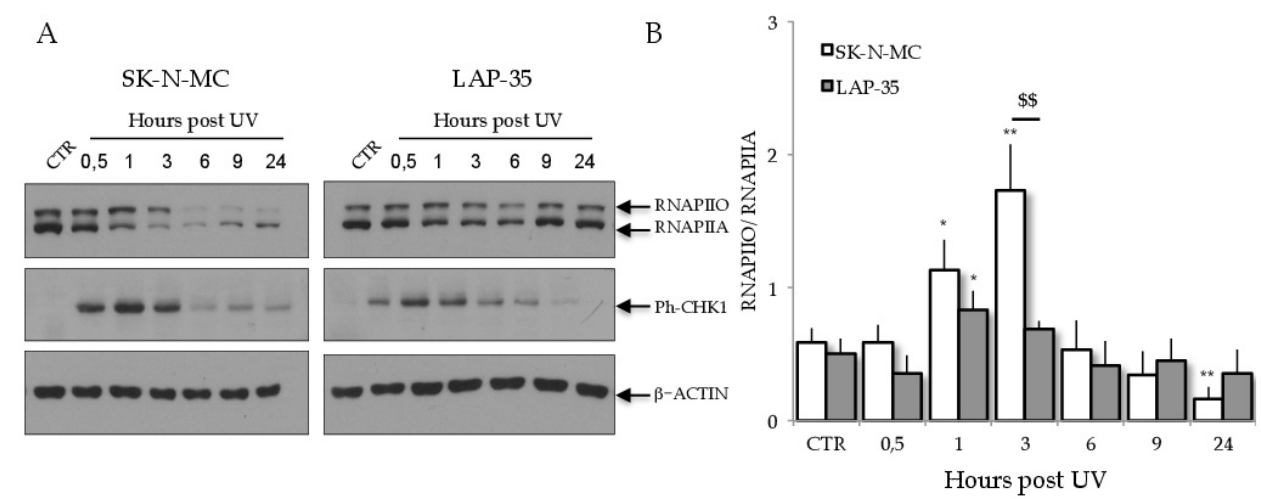

C

$\mathrm{D}$

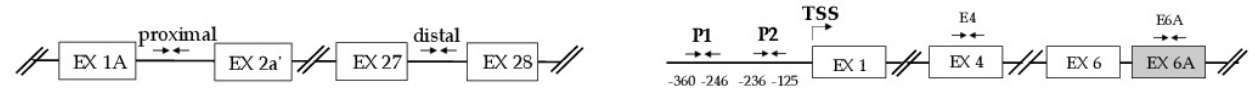

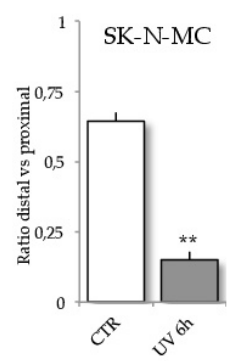

E

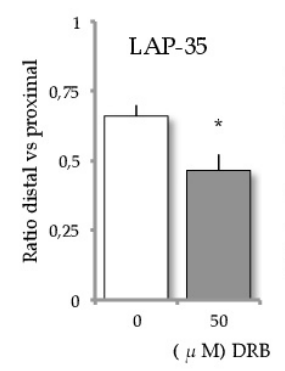

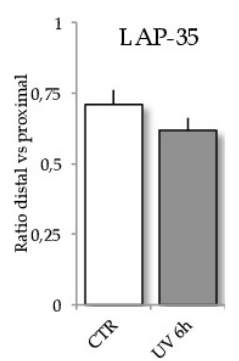

$\mathrm{F}$

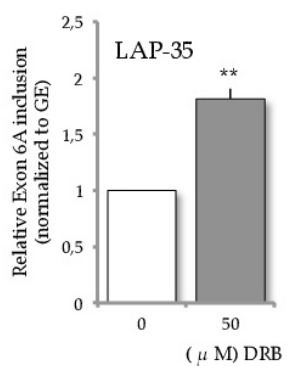

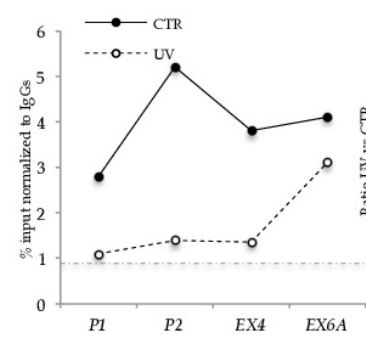

G

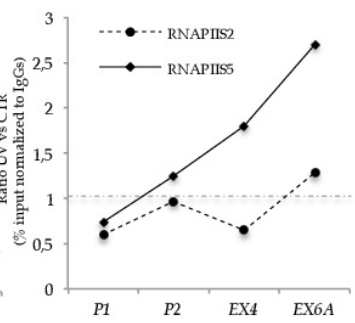

$\mathrm{H}$

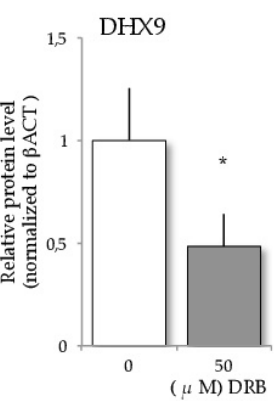

Figure 3: RNAPII dynamics in ES cells upon UV light treatment. A. Western blot analysis of RNAPII, Phospho-CHK1 (in order to control the activation of a protein kinase-signaling cascade initiated by ATM and ATR protein kinases upon DNA damage) and $\beta$-ACTIN expression in SK-N-MC and LAP-35 cells after UV light $\left(10 \mathrm{~J} / \mathrm{m}^{2}\right)$ treatment. B. Histograms represent the ratio between the hyperphosphorylated (RNAPIIO) and the hypo-phosphorylated (RNAPIIA) RNAPII from three independent experiments in SK-N-MC (white bars) and LAP-35 (grey bars) cells (mean \pm s.d.). Statistical analysis was performed by Student's $t$-test: ${ }^{*} p<0.05,{ }^{* *} p<0.01$, for treated vs untreated; $\$ \$ p<0.01$ for SK-N-MC vs LAP-35 cells. C. In the upper part, scheme of DHX9 transcription unit showing the primers (arrows) designed to amplify proximal and distal amplicons in RT-qPCR analysis. Histograms represent RNAPII processivity, determined as a ratio between the distal and proximal amplicons in DHX9 pre-mRNA in control (white) and UV (10 J/m²) treated (grey) SK-N-MC (on the left) and LAP-35 cells (on the right). D. ChIP analysis of RNAPII occupancy on DHX9 transcription unit in normal condition and upon UV light treatment. In the upper part, scheme of DHX9 transcription unit showing the primers (arrows) designed to amplify the promoter and amplicons in the constitutive exon 4 and in the alternative exon 6A. On the left, chart represents total RNAPII binding as input percentage normalized to IgGs in normal conditions (continuous line) and at 6 hours upon UV light treatment (dashed line). On the right, chart represents PhosphoSer2-RNAPII (continuous line) and PhosphoSer5-RNAPII (dashed line) binding to DHX9 transcription unit. Binding is expressed as input percentage of UV versus CTR. E-F. RNAPII processivity and relative exon 6A inclusion after 12-hours $50 \mu \mathrm{M}$-DRB treatment in LAP-35 cells. Exon 6A inclusion rises and RNAPII processivity decreases in DRB-treated LAP-35. Histograms represent mean \pm s.d. from three independent biological replicas (treated vs untreated: ${ }^{*} p<0.05,{ }^{* *} p<0.01$ ). G. Western blot analysis of DHX9 and $\beta$-ACTIN expression in extracts $(10 \mu \mathrm{g})$ from LAP-35 treated with $50 \mu \mathrm{M}$ DRB (12 hours). H. Histograms represent quantification of DHX9 protein normalized to $\beta$-ACTIN $(n=3$; mean \pm s.d.; $* * p<0.01)$. 
downregulated in both cell lines (Figure 4A). These results show that UV light irradiation induces widespread downregulation of EWS-FLI1 target genes in SK-N-MC cells.

Since DHX9 enhances EWS-FLI1 transcriptional activity, its downregulation upon UV light irradiation may directly affect EWS-FLI1 function. To test if UV light irradiation affected the recruitment of EWS-FLI1 to target genes, we performed chromatin immunoprecipitation (ChIP) experiments in both SK-N-MC and LAP-35 cells. Recruitment of EWS-FLI1 to ID2, $C C N D 1$, and $c-M Y C$ promoters was strongly impaired by UV light treatment in SK-N-MC, but not in LAP-35 cells where we actually observed the opposite trend (Figure 4B), even though this enrichment did not result in increased expression of the target genes (Figure 4A). Accordingly, the interaction between DHX9 and EWS-FLI1 was not affected by UV light treatment in LAP-35 cells (Figure S5C, S5D).

These results suggest that downregulation of DHX9 expression induced by UV light irradiation in SK-N-MC cells impairs recruitment of EWS-FLI1 on the promoters of its target genes.

\section{DHX9 is involved in modulation of EWS-FLI1 target genes}

To endorse the hypothesis that expression of EWS-FLI1 target genes in response to UV irradiation was linked to $D H X 9$ expression in ES cells, we knocked down $D H X 9$ in LAP-35 cells. Reduction of $D H X 9$ expression was confirmed both at the RNA (52\% reduction versus scrambled; Figure $5 \mathrm{~A})$ and protein level (64\% reduction versus scrambled; Figure 5B, 5C). RT-qPCR analysis revealed that $D H X 9$ knockdown affected expression of EWS-FLI1 target genes in LAP-35 cells, leading to down-regulation of ID2 (20\%), CCND1 (46\%) and c-MYC (40\%) mRNA levels (Figure 5D). Importantly, RT-qPCR and western blot analysis revealed that knockdown of $D H X 9$ expression did not affect EWS-FLI1 expression in LAP-35 cells (Figure 5B, 5C), ruling out that the effect on EWSFLI1 target genes was indirectly due to modulation of its expression. Furthermore, knockdown of $D H X 9$ in SK-N-MC cells recapitulated the effect of UV treatment on the expression of EWS-FLI1 target genes (Figure 5E). No major changes in RNAPII phosphorylation were detected upon DHX9 knockdown in SK-N-MC cells (Figure S4E, S4F), suggesting that DHX9 acts directly on EWS-FLI1.

To further corroborate our hypothesis, we analyzed the recruitment of EWS-FLI1 and RNAPII on EWS-FLI1 target genes by ChIP analysis in LAP-35 cells. DHX9 knockdown strongly impaired recruitment of both EWSFLI1 and RNAPII on the promoters of EWS-FLI1 target genes (Figure 5F, 5G).
These data suggest that reduced expression of DHX9 induced by low doses of UV light irradiation contributes to downregulation of EWS-FLI1 target genes in ES cells (Figure 5H).

\section{DHX9 confers UV light resistance to ES cells}

DHX9 expression is necessary for ES cells to proliferate $[17,35]$. To test whether its expression is also relevant for ES cell survival in response to UV light irradiation, we overexpressed recombinant GFP or GFP-DHX9 in SK-N-MC cells. PI staining at 0, 24 and 48 hours after UV light treatment revealed that DHX9overexpressing SK-N-MC cells are significantly more resistant to UV light treatment (Figure 6A). This result was also supported by clonogenic assays. SK-N-MC cells were transfected with either GFP or GFP-DHX9 and GFP positive cells were isolated by cell sorting before the treatment with low doses UV light. Up-regulation of DHX9 in SK-N-MC cells significantly enhanced clonogenic activity in response to UV light treatment (Figure 6B).

To further prove the involvement of DHX9 in the resistance of ES cells to UV irradiation, we knocked down its expression in LAP-35 cells. In line with our hypothesis, knockdown of DHX9 in LAP-35 cells reduced resistance to UV light treatment, as indicated by the higher number of PI-positive cells at 24-48 hours after irradiation (Figure 6C) and the reduced number of colonies observed in clonogenic assays (Figure 6D). Interestingly, transfection of recombinant GFP-DHX9 in HeLa cells, which do not express the EWS-FLI1 oncogene, did not improve resistance to UV light irradiation (Figure S6A, S6B), indicating that DHX9 function is mediated by an ETS transcription factor (i.e. EWS-FLI1).

These results strongly indicate that DHX9 expression confers resistance to UV light irradiation to ES cells and suggest that downregulation of DHX9 expression or activity could be instrumental to sensitize ES cells to genotoxic stress.

\section{Genotoxic stress affects $\mathrm{DHX} 9$ alternative splicing}

In order to test whether other genotoxic agents affected SK-N-MC cells viability, we performed clonogenic and cell viability assays using increasing concentrations of etoposide (Eto), 5-fluorouracile (5FU) and cisplatin (CIS). These drugs are widely used in chemotherapy for their antineoplastic properties [36]. Etoposide is a topoisomerase II inhibitor, 5-fluorouracile is a pyrimidine analog, and cisplatin is an alkylating agent; all of them induce DNA damage and cell death 


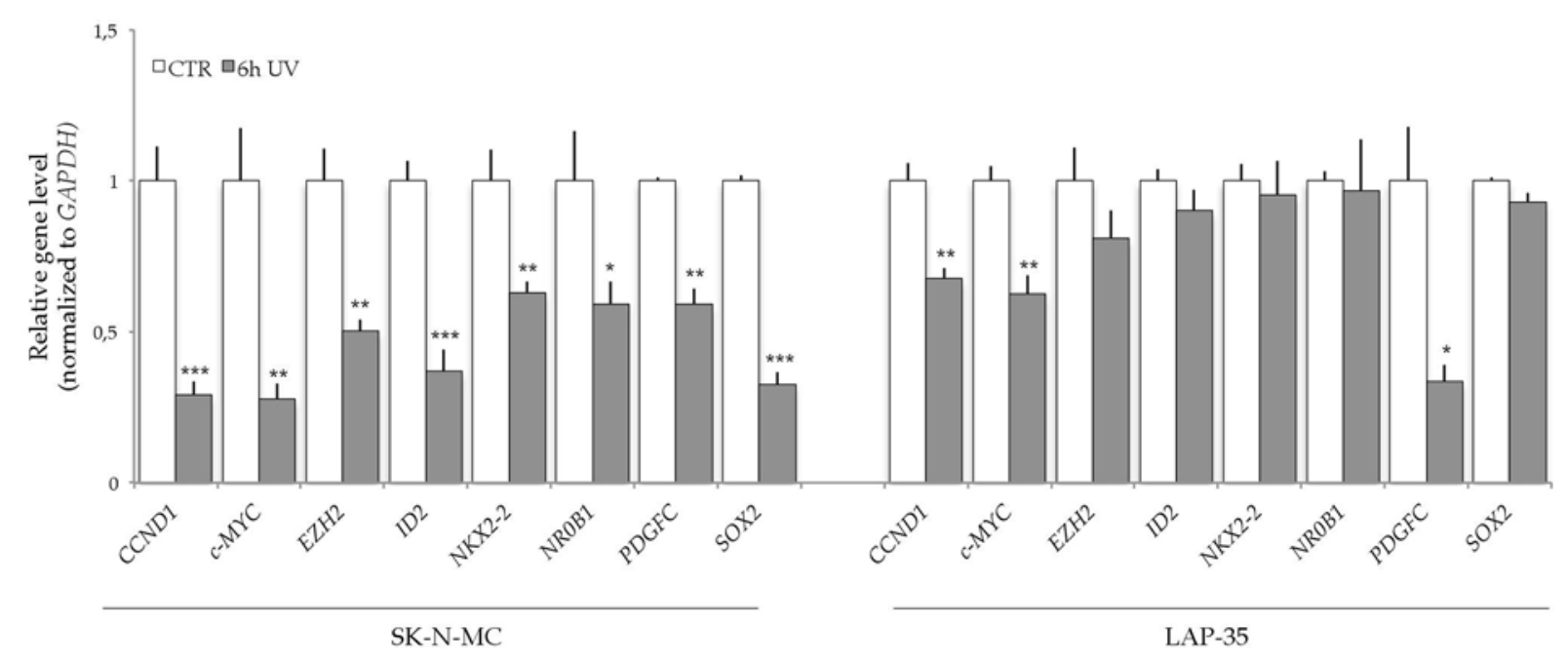

B

\section{ChIP FLI1}

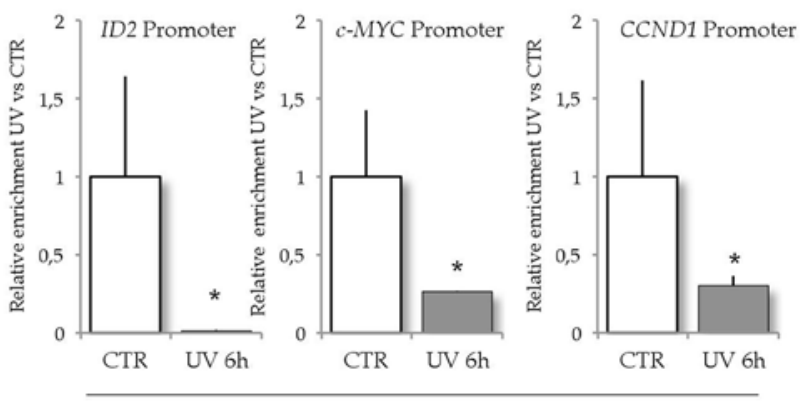

SK-N-MC
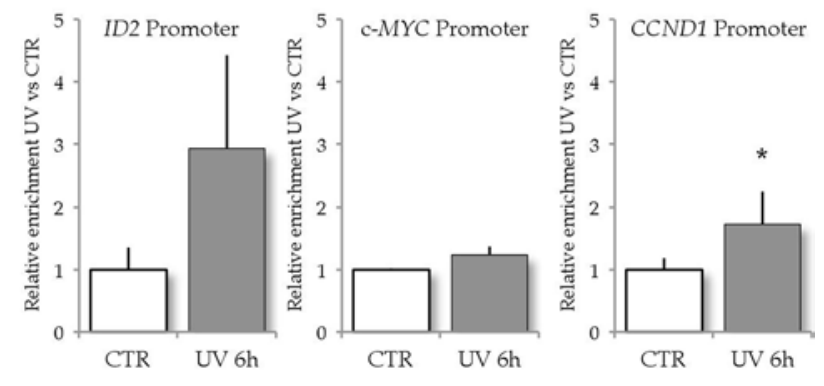

LAP-35

Figure 4: UV light treatment down-regulates EWS-FLI1 target genes in SK-N-MC cells. A. Histograms represent RT-qPCR analysis of ID2, c-MYC, CCND1 EZH2, NKX2-2, NROB1, PDGFC, SOX2 expression in control (white bar) or UV-treated (gray bar) LAP-35 and SK-N-MC cells. GE values of are normalized to GAPDH expression $(n=3$; mean \pm s.d.; treated vs untreated $* p<0.05$, $\left.{ }^{* *} p<0.01, * * * p<0.001\right)$. B. Association of EWS-FLI1 to the promoters of ID2, CCND1 and $c-M Y C$ genes. qPCR analysis of EWS-FLI1 ChIP signals for SK-N-MC and LAP-35 cells with or without UV treatment. Histograms represent relative fold enrichment of EWS-FLI1 normalized versus the IgGs content $(n=3$; mean \pm s.d.; $* p<0.05, * * p<0.01, * * * p<0.001)$.

[36]. We found that etoposide treatment specifically impaired SK-N-MC cells proliferation and clonogenicity, while 5-fluorouracile and cisplatin had milder effect on cell viability (Figure 7A-7C). Importantly, western blot analysis documented that etoposide was also the only chemotherapeutic drug affecting RNAPII phosphorylation (Figure 7D, 7E), in a manner similar to what observed at later time points after UV irradiation (Figure 3A). Furthermore, etoposide treatment also enhanced $D H X 9$ exon 6A inclusion (Figure 7F), thus decreasing DHX9 protein expression (Figure 7D-7G) similarly to UV light irradiation.

These results document that etoposide treatment parallels the effects of UV light irradiation on DHX9 alternative splicing, RNAPII phosphorylation and SK-N-MC viability. Accordingly, etoposide treatment displayed a milder effect on LAP35 cells (Figure S6C). On the other hand, treatment of both LAP-35 and SK-N-MC cells with the YK-4-279 inhibitor, which inhibits DHX9EWS-FLI1 interaction, affects viability and proliferation of both ES cell lines (Figure S6D, S6E).

\section{DISCUSSION}

The study presented here identifies DHX9 as a novel regulator of ES cells sensitivity to UV light irradiation. We provide evidence that DNA damage regulates $D H X 9$ expression by AS through the inclusion of a new PTCcontaining exon and consequent targeting to NMD. UV light regulates this event in a CTD-dependent manner by slowing down the elongation rate of RNAPII and promoting the inclusion of the alternative exon $6 \mathrm{~A}$ in DHX9 pre-mRNA. Furthermore, we show that changes in expression of DHX9 modify the sensitivity to UV 
A

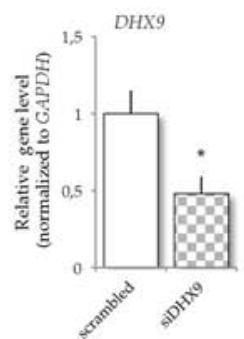

D
B

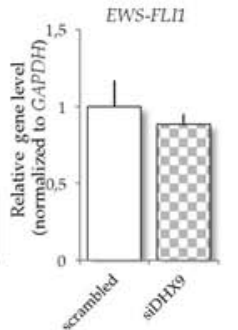

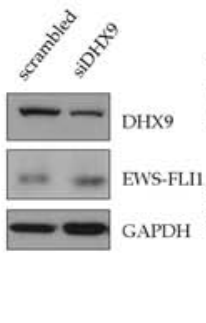

E
C
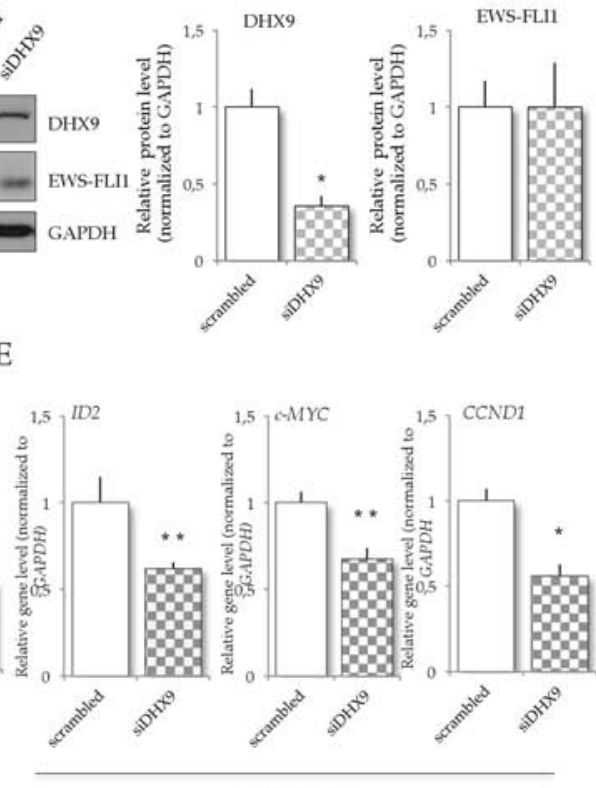

G

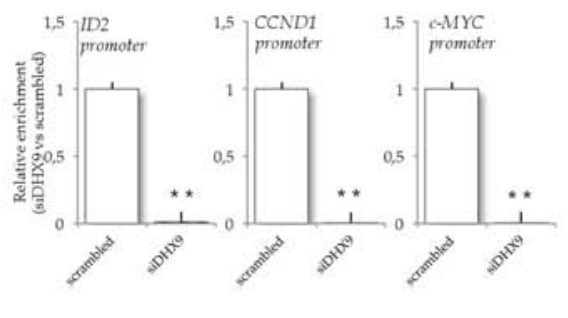

ChIP RNAPII
SK-N-MC

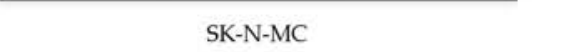

$\mathrm{H}$

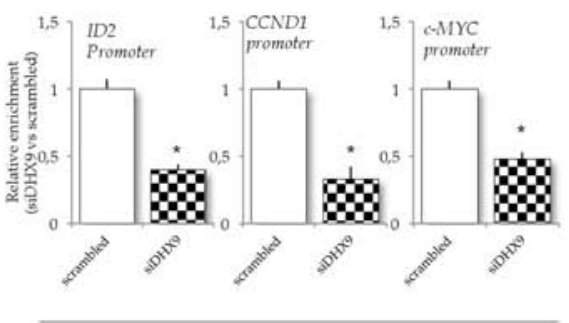

ChIP FLI1 
A

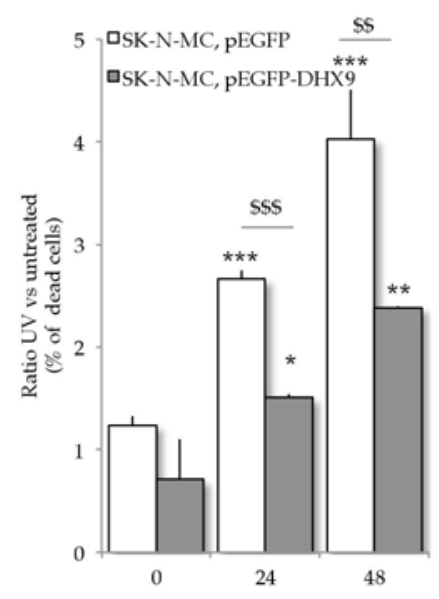

C

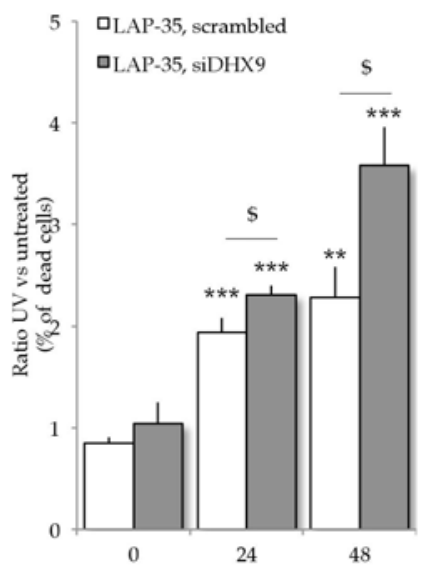

B

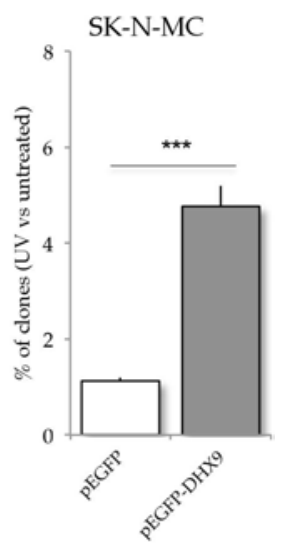

D

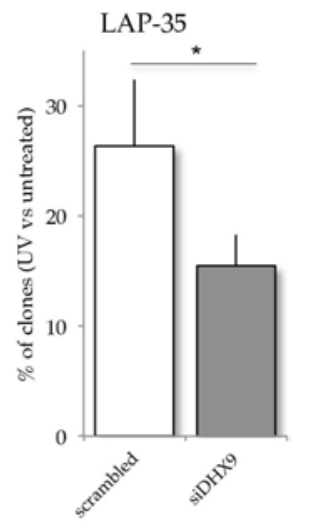

Figure 6: DHX9 expression affects ES resistance to UV light irradiation. A. SK-N-MC cells were transfected with either pEGFP or pEGFP-DHX9 constructs and exposed to $10 \mathrm{~J} / \mathrm{m}^{2} \mathrm{UV}$ light 48 hours after transfection. Cell death was detected at 0,24 and 48 hours after the UV treatment by PI staining and flow cytometry analysis; histograms represent the ratio of UV treated vs untreated PI positive cells in the GFP population ( $\mathrm{n}=3$; mean \pm s.d.; ${ }^{*} p<0.05,{ }^{* *} p<0.01,{ }^{* * *} p<0.001$ for treated vs untreated; and: $\$ p<0.05$, $\$ \$ p<0.01, \$ \$ \$ p<0.001$ for pEGFP vs pEGFP-DHX9). B. GFP-positive SK-N-MC cells transfected as in (A) were sorted and plated (2000 cells per 10-mm plate) in IMDM complete medium. 12 days after $10 \mathrm{~J} / \mathrm{m}^{2} \mathrm{UV}$-light treatment cells were scored for clonogenic activity. Histograms represent the percentage of colonies formed after UV treatment versus untreated carried out on pEGFP (white) or pEGFP-DHX9 (gray) $(n=3$; mean \pm s.d.; pEGFP vs pEGFP-DHX9: $* * * p<0.001)$. C. LAP-35 cells were transfected with either scrambled or siDHX9 oligonucleotides and exposed to $10 \mathrm{~J} / \mathrm{m}^{2} \mathrm{UV}$ light 48 hours after transfection. Cell death was detected at 0,24 and 48 hours after treatment by PI staining and flow cytometry analysis; histograms represent the ratio of UV treated vs untreated PI positive cells $(n=3$, mean \pm s.d.; treated vs untreated: ${ }^{*} p<0.05,{ }^{* *} p<0.01,{ }^{* * *} p<0.001$, for treated vs untreated; and: $\$ p<0.05, \$ \$ p<0.01, \$ \$ \$ \boldsymbol{p}<0.001$ for scrambled vs siDHX9. D. LAP-35 cells were transfected with either scrambled or siDHX9 oligonucleotides together with pEGFP plasmid; GFP positive cells were sorted and plated as above. 12 days after $10 \mathrm{~J} / \mathrm{m}^{2} \mathrm{UV}$ light treatment, cells were scored for clonogenic activity. Histograms represent the percentage of colonies after UV treatment versus untreated cells carried out on scrambled (white bars) or siDHX9 (gray) LAP-35 cells $\left(n=3\right.$, mean \pm s.d.; scrambled vs siDHX9: $\left.{ }^{*} p<0.05\right)$.

irradiation and that DHX9 is a novel pro-survival factor in ES cells. Notably, among other chemotherapeutic agents tested, etoposide, the only one that efficiently suppressed ES cell growth, was also the only agent affecting RNAPII phosphorylation and exon 6A splicing. These results identify modulation of $D H X 9$ splicing as a new potential tool to enhance ES cell sensitivity to genotoxic stresses.
Cancer cells are characterized by alteration of gene expression both at the level of transcription and mRNA processing, giving rise to many splice variants specifically associated with cancer [15]. In particular, AS plays a key role in the cellular response to stress and DNA damage, influencing the response to chemotherapeutic agents $[7,8]$. Notably, splicing factors and components 

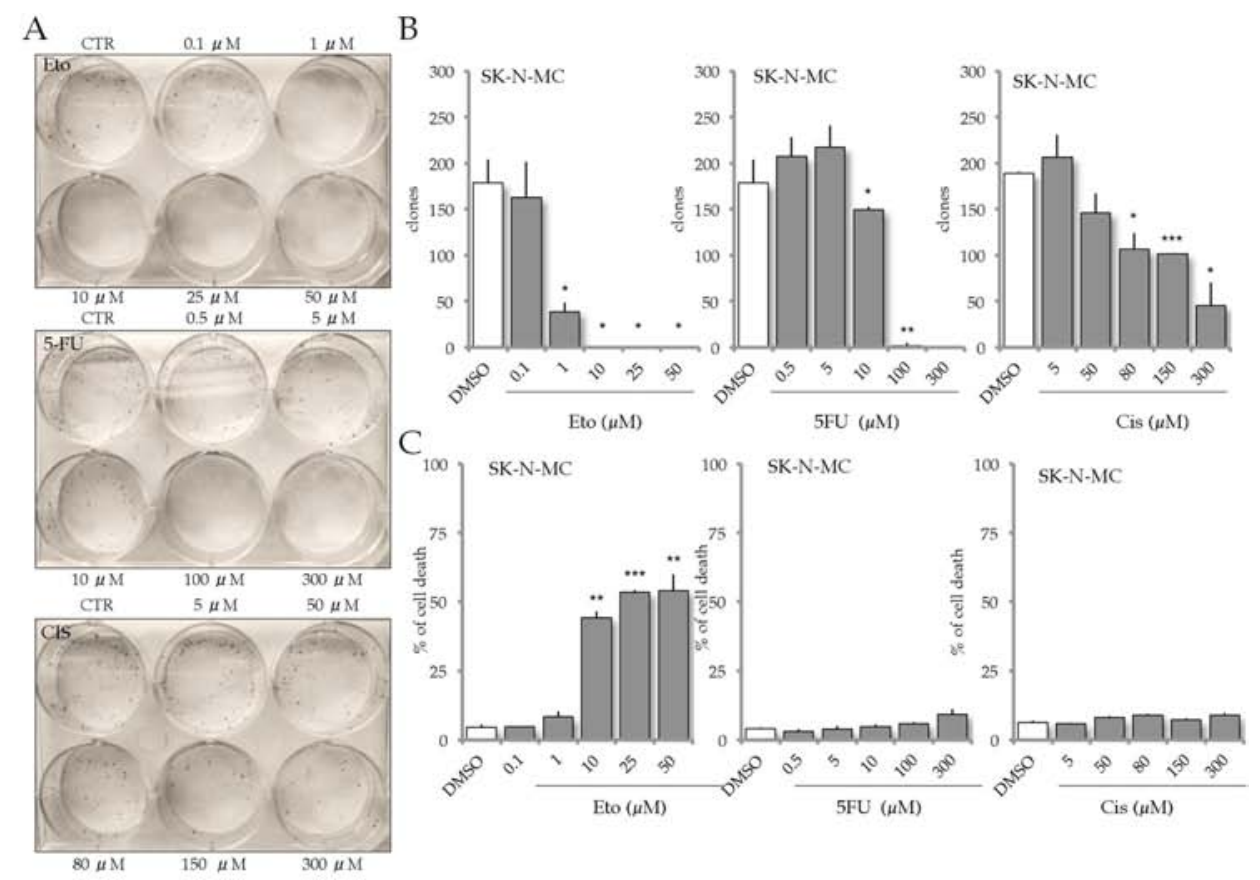

D

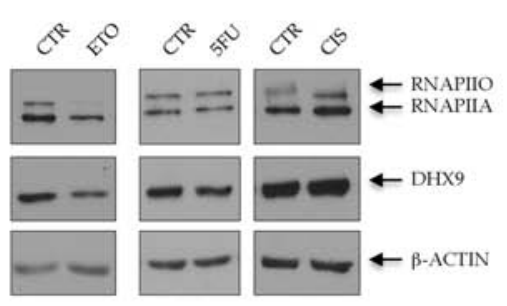

F
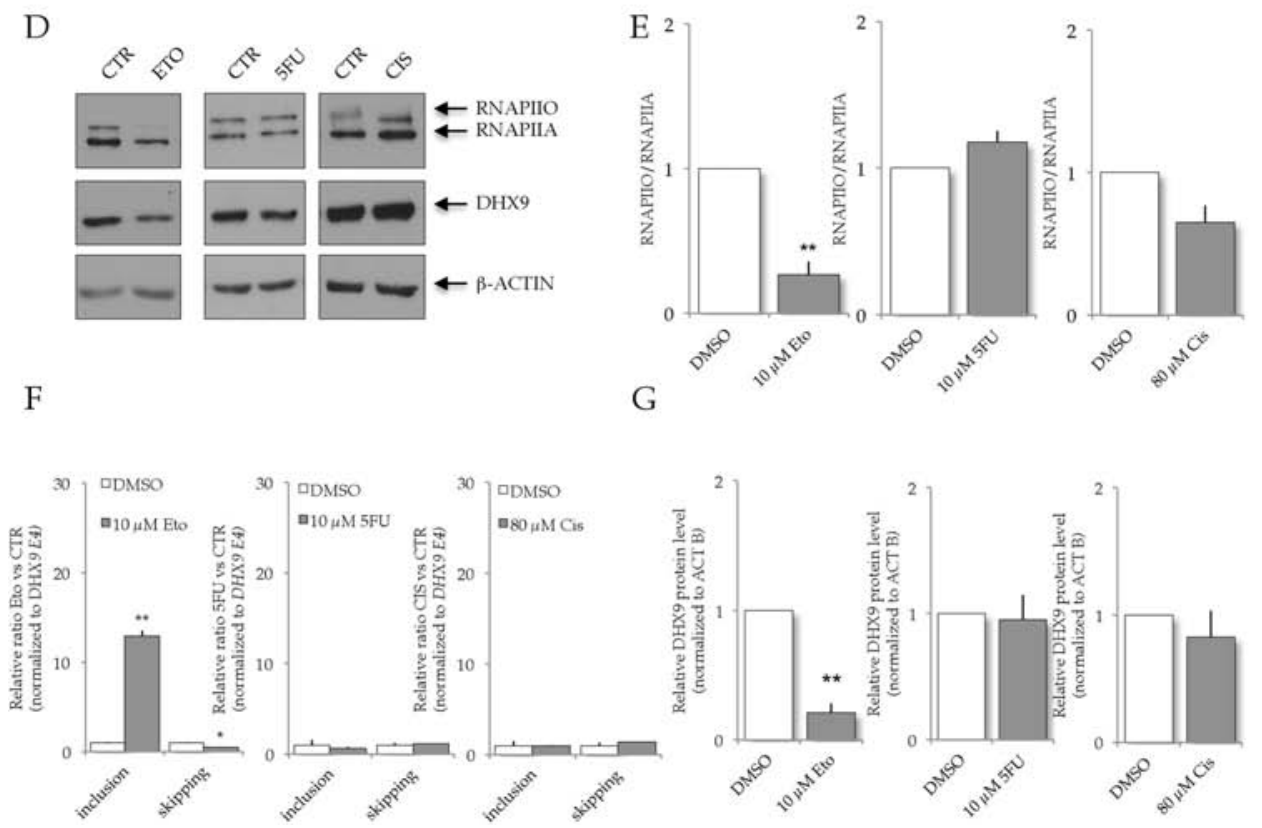

Figure 7: Etoposide treatment affects DHX9 expression and ES sensitivity. A. Representative images of clonogenic assays of SK-N-MC cells upon treatment with different concentration of etoposide (Eto; from 0,1 to $50 \mu \mathrm{M})$, 5 -fluorouracile (5-FU; from 0,5 to $300 \mu \mathrm{M})$, and cisplatin (CIS; from 5 to $300 \mu \mathrm{M})$. B. Histograms represent colony numbers $(n=3$; mean \pm s.d.) carried out on SK-N-MC treated with Eto, 5-FU and CIS (grey bars) versus DMSO treatment (white bars). C. Propidium Iodide (PI) viability assay; the decrease in viability was expressed as relative percentage of dead cells in treated (grey bars) versus control (white bars, DMSO) cells after 16 hours of Eto, 5FU and CIS treatment. In all panels statistical analysis was performed by Student's $t$-test: ${ }^{*} p<0.05,{ }^{* *} p<0.01,{ }^{* * *} p<0.001$. D. Western blot analysis of RNAPII, DHX9 and $\beta$-ACTIN expression in SK-N-MC after 16 hours treatment with DMSO, Eto $(10 \mu \mathrm{M}), 5$-FU (10 $\mu \mathrm{M})$, and CIS $(80 \mu \mathrm{M}) .10 \mu \mathrm{g}$ of extracts were loaded in each lane of a $6 \%$ SDS PAGE. E. Histograms represent the ratio between the hyperphosphorylated (RNAPIIO) and the hypo-phosphorylated (RNAPIIA) RNAPII from three independent experiments in SK-N-MC after 16 hours treatment with DMSO (white bars) or Eto $(10 \mu \mathrm{M}), 5 \mathrm{FU}(10 \mu \mathrm{M})$, and CIS $(80 \mu \mathrm{M})$ (grey bars), as in (D) (mean \pm s.d.). Statistical analysis was performed by Student's $t$-test: $* p<0.05, * * p<0.01, * * * p<0.001$ F. RT-qPCR analysis to monitor DHX9 AS upon Eto $(10 \mu \mathrm{M}), 5-\mathrm{FU}(10 \mu \mathrm{M})$, and CIS $(80 \mu \mathrm{M})$ treatment. Histograms represent levels of expression of DHX9 exon 6A normalized to a constitutive exon (E4) in SK-N-MC cells. G. Histograms represent DHX9 expression from three independent experiments in SK-N-MC treated for 16 hours with DMSO (white bars) or Eto $(10 \mu \mathrm{M}), 5-\mathrm{FU}(10 \mu \mathrm{M})$, and CIS $(80 \mu \mathrm{M})$ (grey bars), as in (D) Statistical analysis was performed by Student's $t$-test: $* p<0.05, * * p<0.01, * * * p<0.001$. 
of the transcription machinery, including RNAPII itself, can be targets of intracellular cascades that link changes in splicing patterns to DNA damage signals [14]. For instance, UV light treatment triggers the inhibition of transcription initiation by RNAPII [23]. Furthermore, the BRCA-1/BARD-1 complex ubiquitinates the phosphorylated form of RNAPII, inducing its degradation by the proteasome [37]. Thus, the timing and dynamics of RNAPII phosphorylation and degradation take part to the fine-tuned regulation of AS upon UV light irradiation ([14]; present study). In this regard, we found that regulation of RNAPII correlates with the sensitivity of ES cells to UV irradiation. RNAPII is strongly downregulated in SK-N-MC cells 3-6 hours after UV light treatment, whereas it was only slightly reduced in LAP-35 cells (Figure 3A, 3B). Moreover, the phosphorylation status of the CTD (RNAPIIO) was regulated differently in the two cell lines; RNAPIIO peaked at three hours upon UV light treatment in SK-N-MC cells, and then it was strongly reduced. On the contrary, the phosphorylation status of the CTD was only slightly affected in LAP-35 cells under these conditions (Figure 3A, 3B). In line with the effects on RNAPII dynamics, larger changes in gene expression and AS were observed in SK-N-MC than in LAP-35 cells, suggesting that the two events triggered by UV irradiation were mechanistically linked.

To investigate the mechanism underlying the different sensitivity of ES cells to UV irradiation, we searched for genes that were differentially regulated in SK-N-MC and LAP-35 cells. Among them, DHX9 was specifically affected both at gene expression and AS upon UV light irradiation only in SK-N-MC. Interestingly, UV light induced the inclusion of a PTC-containing exon in the DHX9 transcript (the alternative exon 6A), thus targeting the corresponding transcript to NMD and contributing to $D H X 9$ down-regulation. As a consequence, in response to UV light treatment, the DHX9 protein is diminished in SK-N-MC but not in LAP-35 cells (Figure 2). This observation is in line with other studies showing that exons sensitive to RNAPII modulation often introduce PTCs that elicit NMD of the spliced mRNAs [14, 33, 38].

Mechanistically, inclusion of exon 6A in $D H X 9$ premRNA is linked with modulation of RNAPII elongation rate by UV treatment. We found that RNAPII processivity was significantly reduced in SK-N-MC cells but not in LAP-35 cells (Figure 3D), possibly due to the stronger effect of UV light on RNAPII phosphorylation in the former cells. Indeed, strong phosphorylation of RNAPII was previously shown to reduce its elongation rate in cells exposed to genotoxic stress [14]. In line with this concept, we found that treatment of LAP-35 cells with the pTEFB inhibitor DRB artificially affected RNAPII processivity and recapitulated inclusion of the alternative exon $6 \mathrm{~A}$ of DHX9 (Figure 3F), reducing DHX9 protein expression (Figure $3 \mathrm{G}, 3 \mathrm{H}$ ). Thus, our results prove that factors affecting RNAPII elongation rate (UV irradiation and
DRB) regulate $\mathrm{DH} X 9 \mathrm{AS}$ and expression by controlling the inclusion of an alternative PTC-containing exon in ES cells. Nevertheless, we cannot exclude that UV light irradiation, or etopside treatment, may also induce the expression or activity of some splicing factors, thus contributing to exon 6A inclusion. Given the importance of DHX9 in cancer cells resistance and proliferation, future work will investigate this issue.

DHX9 is a member of the DEXH family of RNA helicases [39], which play important roles in several aspects of RNA metabolism [40]. Homozygous Dhx 9 mutation in mice determines early embryonic lethality [41], suggesting that the $D H X 9$ gene is essential for viability. Moreover, through its helicase activity DHX9 binds to and resolves mutagenic intra-molecular triplex structures [42, 43], preventing genomic instability and assisting the maintenance of DNA integrity in the replication, recombination, and repair processes. In ES cells, DHX9 forms a complex with EWS-FLI1 and modulates EWS-FLI1-dependent transcription [17]. Thus, DHX9 could be a functional partner for EWS-FLI1 by enhancing engagement of the transcriptional machinery at responsive promoters and inducing local changes in chromatin structure and DNA unwinding. We found that the reduction of DHX9 expression in SK-N-MC cells elicited by treatment with UV light correlates with downregulation of EWS-FLI1 target genes, suggesting that it is physiologically relevant. Moreover, EWS-FLI1 recruitment to the promoter of its target genes is repressed upon UV treatment only in SK-N-MC cells, where DHX9 expression is downregulated, but not in LAP-35 cells, where DHX9 protein remains high. Consistent with our model is also the observation that $D H X 9$ knockdown in LAP-35 and in SK-N-MC cells, to similar levels of those obtained by UV irradiation in SK-N-MC cells, is sufficient to repress the expression of ID2, $c-M Y C$ and $C C N D 1$ (Figure 5), regardless of the UV treatment. These results strongly suggest that downregulation of EWS-FLI1 target genes induced by UV light irradiation in SK-N-MC cells is due to reduced expression of DHX9.

The functional interaction between EWS-FLI1 and DHX9 was previously shown to support anchorageindependent growth [17]. In this study, we found that DHX9 expression confers resistance to UV irradiation in ES cells. Indeed, overexpression of recombinant DHX9 makes SK-N-MC cells significantly more resistant to UV light treatment. Conversely, knockdown of endogenous DHX9 renders LAP-35 cells more sensitive to UV light treatment. Since overexpression of DHX9 in HeLa cells did not improve cell survival to UV irradiation, this phenomenon appears to be specific for ES cells. Collectively, these results suggest that DHX9 downregulation could be instrumental to sensitize ES cells to genotoxic stress. In line with this concept, an RNAi screen recently uncovered $D H X 9$ as a key target gene to sensitize lymphomas to chemotherapeutic 
treatment [44]. Although DHX9 might play a direct role in DNA repair and genome maintenance through its interaction with the Werner syndrome helicase WRN [45] and BRCA1 [46], our results suggest that it may also participate to this process by enhancing the expression of oncogenic EWS-FLI1 target genes. On the other hand, it is also possible that, in addition to $D H X 9$, other genes differentially regulated in the two ES cell lines are involved in the different sensitivity to genotoxic stress. For example, our microarray analysis revealed that FOS gene is specifically upregulated in LAP-35 cells but not in SK-N-MC cells. Notably, cells deficient in $c-F o s$ are hypersensitive to ultraviolet (UV-C) light and mouse embryonic fibroblasts fos $^{-/}-$are defective in the repair of UV-C induced DNA lesions [47].

ES tumors initially respond well to chemotherapy, but $40 \%$ of patients later develop recurrent disease and the majority of them die within 5 years, despite high-dose chemotherapy [48]. Down-regulation of EWS-FLI1 by siRNA approaches resulted in prolonged survival of ES xenograft-bearing mice [49], but this approach currently lacks clinical translation [50]. Recently, a small-molecule targeting EWS-FLI1-DHX9 interaction has been proposed as a strategy to inhibit EWS-FLI1-mediated transcription [35]. Here we provide evidence that genotoxic stresses causing downregulation of DHX9 expression efficiently reduce ES cell growth. Moreover, we have identified a $D H X 9$ splicing isoform induced by UV light treatment that leads to DHX9 down-regulation in ES cells. Since DHX9-EWSFLI1 functional interaction represents a good opportunity for clinical intervention, our data indicate a novel strategy for targeting EWS-FLI1 oncogene activity through the modulation of DHX9 expression or activity. In this scenario, the development of antisense oligonucleotides recruiting the spliceosomal complex to the alternative exon $6 \mathrm{~A}$ in $\mathrm{DH} X 9$ could be instrumental to drive DHX9 downregulation in ES cells and might provide a valuable additional therapy for the treatment of ES.

\section{MATERIALS AND METHODS}

\section{Cell culture and treatments}

SK-N-MC and LAP-35 Ewing Sarcoma cells were a generous gift from Drs F. Moretti and K. Scotlandi, respectively. Both cell lines were cultured in IMDM medium supplemented with $10 \%$ fetal bovine serum, penicillin and streptomycin (Gibco), and maintained at $37^{\circ} \mathrm{C}$ in humidified $5 \% \mathrm{CO}_{2}$ atmosphere. For treatment, cells were plated at 50\%-60\% confluence $16 \mathrm{~h}$ before UV light irradiation (either 10 or $40 \mathrm{~J} / \mathrm{m}^{2}$ ). Fresh medium was immediately added after the treatment and the cells were harvested at different times during the recovery, as indicated. For drug treatment, ES cells were treated 16 hours with DMSO or etoposide, 5-fluorouracile, and cisplatin at different concentration, as indicated.

\section{Splicing-sensitive microarrays}

RNA from three biological replicates of control or UV irradiated Ewing Sarcoma cells (either SK-N-MC or LAP-35) and HEP3B cells collected 6 hours after the treatment was purified using RNeasy Mini kit (Qiagen) and digested with DNase RNase free (Qiagen). cDNA and Cy5-Cy3 labelled cRNA were generated from the total RNA using the Agilent Low RNA Input Fluorescent Linear Amplification kit. $8 \mu \mathrm{g}$ of each cRNA were used for the hybridization with the arrays (Agilent In situ hybridization kit plus). After hybridization, arrays were washed, and scanned images analyzed as previously described [14]. Three biological replicates were hybridized, with both direct and dyereversal hybridizations. General gene expression values represent the average of $\log 2$ ratios for all the probes of a locus. Statistical analyses were carried out with Linear Models for Microarray Data (Limma; Bioconductor Project) [51]. The background correction method used in the analysis was Normexp [52]. Locally weighted linear regression (LOWESS) analysis was used as a normalization method [53].

The cutoff considered was fold change $>|1,3|$ for gene expression changes and fold change $>|1,4|$; (Z-SCORE $>3$; $p$-value $<0,01$ ) for alternative splicing changes. The data were deposited in GEO database with the accession number GSE59889.

\section{Transfection experiments}

For transfections, cells were plated in 35-mm dishes and transfected with siRNAs (Sigma-Aldrich) at final concentration of $100 \mathrm{nM}$ using Lipofectamine RNAiMax reagent (Invitrogen) according to the manufacturer's instructions. The $D H X 9$ and UPF 1 siRNA and scrambled sequences are listed in Supplementary Table 2. For over-expression experiments, transfections were performed with $1 \mu \mathrm{g}$ of appropriate constructs (pEGFP or pEGFP-DHX9) using lipofectamine 2000 (Invitrogen). $48 \mathrm{~h}$ after transfections, cells were treated and collected at the indicated time points for RNA or protein analyses.

\section{Isolation of total RNA and RT-qPCR}

Total RNA was extracted by using TriPure Isolation Reagent (Roche) according to the manufacturer's instructions and subjected to DNase digestion (Roche). First-strand cDNA was obtained from $1 \mu \mathrm{g}$ of RNA using random hexamer and M-MLV-Reverse Transcriptase (Promega, Italy). Synthesized cDNA corresponding to 25 ng total RNA was used for conventional- (GoTaq DNA Polymerase, Promega) or quantitative-PCR (SYBR Green Master Mix for Light-Cycler 480, Roche), according to manufacturer's instructions. Primers used for RT-qPCR are listed in the Supplementary Table 2. 


\section{Protein extraction and western blotting analyses}

Total protein extracts were prepared using lysis buffer $(100 \mathrm{mM} \mathrm{NaCl}, 10 \mathrm{mM} \mathrm{MgCl}, 30 \mathrm{mM}$ Tris-HCl, $\mathrm{pH} 7.5,10 \%$ glycerol, $1 \mathrm{mM}$ dithiothreitol, $10 \mathrm{mM}$ $\beta$-glycerophosphate, $0.5 \mathrm{mM} \mathrm{Na} \mathrm{VO}_{4}$, and protease inhibitor cocktail (Sigma-Aldrich) supplemented with $0.5 \%$ Triton X-100. The extracts were sonicated (1 sec at $30 \%$ ), incubated on ice for $10 \mathrm{~min}$. and then centrifuged for $10 \mathrm{~min}$ at $12,000 \mathrm{~g}$ at $4^{\circ} \mathrm{C}$. Protein quantification was performed by Quick Start Bradford Protein Assay (Bio-Rad). Cell extracts were diluted in SDS sample buffer and boiled for $5 \mathrm{~min}$. Proteins $(10-50 \mu \mathrm{g})$ were separated on $6 \%$ or $10 \%$ SDS-PAGE gels and transferred to Hybond-P membranes (GE Healthcare). Membranes were saturated with $5 \%$ non-fat dry milk in phosphatebuffered saline (PBS) containing $0.1 \%$ Tween- 20 for $1 \mathrm{~h}$ at room temperature, and incubated with the following antibodies and dilutions overnight at $4^{\circ} \mathrm{C}$ : anti-DHX9 1:200 (H300, Santa Cruz Biotechnology), anti-FLI-1 1:200 (sc-356 Santa Cruz Biotechnology), antiGAPDH 1:1000 (sc-32233 Santa Cruz Biotechnology), anti-RNAPII 1:250 (N20, Santa Cruz Biotechnology), anti- $\beta$-ACTIN 1:1000 (MAB1501, Merk Millipore), anti-ERK2 1:1000 (Santa Cruz Biotechnology), and phospho-CHK1 1:1000 (Cell Signaling), anti-UPF1 1:500 (Millipore). Secondary anti-mouse or anti-rabbit IgGs conjugated to horseradish peroxidase (Amersham) were incubated with the membranes for $1 \mathrm{~h}$ at room temperature at a 1:10000 dilution in PBS containing $0.1 \%$ Tween-20. Immunostained bands were detected by a chemiluminescent method (Santa Cruz Biotechnology). Densitometric analysis was performed by ImageJ software.

\section{MTS proliferation assay}

Cell proliferation was determined using the Cell Titer A96 3-(4,5-dimethylthiazol-2-yl)-5-(3carboxymethoxyphenyl)-2-(4-sulfophenyl)-2H-

tetrazolium, inner salt (MTS) method according to the manufacturer's instructions (Promega) by plating $5 \times 10^{4}$ cells/well in 96 -well culture plates.

\section{Chromatin immunoprecipitation}

$2 \times 10^{8}$ SK-N-MC and LAP-35 cells were crosslinked with $1 \%(\mathrm{w} / \mathrm{v})$ formaldehyde (Sigma Aldrich) for $15 \mathrm{~min}$ at room temperature, and then formaldehyde was inactivated by the addition of $125 \mathrm{mM}$ glycine (Sigma Aldrich). Cells were washed in cold PBS and lysed to isolate nuclei in a hypotonic buffer containing $5 \mathrm{mM}$ PIPES (pH 8.0), $85 \mathrm{mM} \mathrm{KCl}, \mathrm{NP} 40$ 0.5\%, $1 \mathrm{mM}$ dithiothreitol, $10 \mathrm{mM} \beta$-glycerophosphate, $0.5 \mathrm{mM} \mathrm{Na}_{3} \mathrm{VO}_{4}$, and protease inhibitor cocktail (Sigma-Aldrich). Isolated nuclei were lysed in a buffer containing $1 \%$ SDS, 10 mM EDTA, and $50 \mathrm{mM}$ Tris- $\mathrm{HCl}$ (pH 8.0), $1 \mathrm{mM}$ dithiothreitol, $10 \mathrm{mM}$ $\beta$-glycerophosphate, $0.5 \mathrm{mM} \mathrm{Na} \mathrm{VO}_{4}$, and protease inhibitor cocktail (Sigma-Aldrich) and sonicated with Bioruptor (Dyagenode) 2 fold for 6 min (30 sec sonication and $30 \mathrm{sec}$ pause). Chromatin extracts containing DNA fragments (100 $\mu \mathrm{g} / \mathrm{sample})$ with an average size of $200 \mathrm{bp}$ were pre-cleared overnight and then immunoprecipitated for 3 hours using $2 \mu \mathrm{g}$ of anti-FLI1 (SAB2100822, Sigma Aldrich) or anti-RNAPII (N20, Santa Cruz Biotechnology) or anti RNAPII CTD repeat H14 and H5 (ab24759 and ab24758, Abcam) antibodies and Protein A/G Agarose/ Salmon Sperm DNA (Merck Millipore). Precipitated DNA was extracted and analyzed by qPCR using primers listed in Supplementary Table 2.

\section{Flow cytometry analysis}

Percentage of dead cells was determined by staining with propidium iodide (PI $1 \mu \mathrm{g} / \mathrm{ml}$ eBioscience) and acquisition was performed on flow cytometry (Canto, BD Biosciences). Analysis was performed using FlowJo software. In some experiments GFP positive population of SK-N-MC cells transfected with pEGFP and pEGFPDHX9 constructs, was isolated using a MoFlo high speed cell sorter (Beckman Coulter).

\section{Colony formation assay}

Cell suspensions were plated in $100-\mathrm{mm}$ plates or $35-\mathrm{mm}$ plates at a density of 2000 or 1000 cells/ plate, respectively. After one day, cells were treated (UV $10 \mathrm{~J} / \mathrm{m}^{2}$ ) and incubated at $37^{\circ} \mathrm{C}$ in a humidified atmosphere containing 5\% CO2 for 12 day, replacing medium every two days. At the end of the incubation period, cells were washed with PBS, fixed in methanol for $10 \mathrm{~min}$ at RT and stained $30 \mathrm{~min}$ at room temperature with $0.05 \%$ Crystal Violet in distilled water on a rotating shaker. After staining, cells were washed twice with tap water and air-dried overnight. The next day clones were counted.

\section{5' splice site score calculation}

In order to evaluate the strength of the alternative exon we compared the scores of the $5^{\prime}$ splice sites of the alternative exon $6 \mathrm{~A}$ versus the downstream constitutive exon 7 of $D H X 9$ gene. The score expresses how similar the splice sites fit the consensus sequence. A perfect $5^{\prime}$ splice site AAG|gtaagt would have a score of 12.6. According to these parameters provided by Dr. Michael Zhang, CSHL and statistical data calculated by Dr. Tetsushi Yada using the sequence compilation for the GENIE program, we run the Splice Site Score Calculation (http://rulai.cshl.edu/new_alt_exon_db2/ $\mathrm{HTML} / \mathrm{score} . \mathrm{html}$ ) and we found that the $5^{\prime}$ SpliceSite Score For DHX9 exon 6A (AAG | GTCAGT) is: 5.9 while the score for the downstream constitutive exon (CAG | GTAAGT) is 12.4. Similar results were obtained using the MaxEntScan::score5ss matrix from 
the laboratory of Chris Burge (http://genes.mit.edu/ burgelab/maxent/Xmaxentscan_scoreseq.html).

\section{ACKNOWLEDGMENTS AND FUNDING}

We thank Drs Juan Valcárcel and Claudio Sette for helpful suggestions and critical reading of the manuscript. We are also indebted with Drs K. Scotlandi, F. Moretti, MC Manara and M. Buttarelli for the generous gift of SK-N-MC and LAP-35 ES cell lines, with C. Giorgi for the generous gift of the anti-Upf1 antibody (Millipore), with Dr Elisa De Paola for technical support.

This work was supported by grants from the Associazione Italiana Ricerca sul Cancro (AIRC) (MFAG 11658), from the Association for International Cancer Research (AICR-UK 14-0333) and from the University of Rome "Foro Italico" (RIC052013) to M.P.P.

\section{CONFLICTS OF INTEREST}

The authors declare no conflict of interest.

\section{REFERENCES}

1. Delattre O, Zucman J, Plougastel B, Desmaze C, Melot T, Peter M, Kovar H, Joubert I, de Jong P, Rouleau G, Aurias A, Thomas G. Gene fusion with an ETS DNAbinding domain caused by chromosome translocation in human tumours. Nature. 1992; 359:162-5.

2. Riggi N, Stamenkovic I. The Biology of Ewing sarcoma. Cancer Lett. 2007; 254:1-10.

3. Jedlicka P. Ewing Sarcoma, an enigmatic malignancy of likely progenitor cell origin, driven by transcription factor oncogenic fusions. Int J Clin Exp Pathol. 2010; 3:338-47.

4. Sanchez G, Bittencourt D, Laud K, Barbier J, Delattre O, Auboeuf D, Dutertre M. Alteration of cyclin D1 transcript elongation by a mutated transcription factor up-regulates the oncogenic D1b splice isoform in cancer. Proc Natl Acad Sci U S A. 2008; 105:6004-9.

5. Paronetto MP. Ewing sarcoma protein: a key player in human cancer. Int J Cell Biol. 2013; 2013:642853.

6. Sanchez G, Delattre O, Auboeuf D, Dutertre M. Coupled alteration of transcription and splicing by a single oncogene: boosting the effect on cyclin D1 activity. Cell Cycle. 2008; 7:2299-305.

7. Dutertre M, Sanchez G, De Cian MC, Barbier J, Dardenne E, Gratadou L, Dujardin G, Le Jossic-Corcos C, Corcos L, Auboeuf D. Cotranscriptional exon skipping in the genotoxic stress response. Nat Struct Mol Biol. 2010; 17:1358-66.

8. Paronetto MP, Minana B, Valcarcel J. The Ewing sarcoma protein regulates DNA damage-induced alternative splicing. Mol Cell. 2011; 43:353-68.
9. Paronetto MP, Bernardis I, Volpe E, Bechara E, Sebestyen E, Eyras E, Valcárcel J. Regulation of FAS exon definition and apoptosis by the Ewing sarcoma protein. Cell Rep. 2014; 7:1211-26.

10. Li H, Watford W, Li C, Parmelee A, Bryant MA, Deng C, O'Shea J, Bong Lee S. Ewing sarcoma gene EWS is essential for meiosis and B lymphocyte development. J Clin Invest. 2007; 117:1314-23.

11. Hurov KE, Cotta-Ramusino C, Elledge SJ. A genetic screen identifies the Triple $\mathrm{T}$ complex required for DNA damage signaling and ATM and ATR stability. Genes Dev. 2010; 24:1939-50.

12. O'Connell BC, Adamson B, Lydeard JR, Sowa ME, Ciccia A, Bredemeyer AL, Schlabach M, Gygi SP, Elledge SJ, Harper JW. A genome-wide camptothecin sensitivity screen identifies a mammalian MMS22L-NFKBIL2 complex required for genomic stability. Mol Cell. 2010; 40:645-57.

13. Biamonti G, Caceres JF. Cellular stress and RNA splicing. Trends Biochem Sci. 2009; 34:146-53.

14. Munoz MJ, Perez Santangelo MS, Paronetto MP, de la Mata M, Pelisch F, Boireau S, Glover-Cutter K, Ben-Dov C, Blaustein M, Lozano JJ, Bird G, Bentley D, Bertrand E, Kornblihtt ARl. DNA damage regulates alternative splicing through inhibition of RNA polymerase II elongation. Cell. 2009; 137:708-20.

15. David CJ, Manley JL. Alternative pre-mRNA splicing regulation in cancer: pathways and programs unhinged. Genes Dev. 2010; 24:2343-64.

16. Bonnal S, Vigevani L, Valcarcel J. The spliceosome as a target of novel antitumour drugs. Nat Rev Drug Discov. 2012; 11:847-59.

17. Toretsky JA, Erkizan V, Levenson A, Abaan OD, Parvin JD, Cripe TP, Rice AM, Bong Lee S, Üren A. Oncoprotein EWS-FLI1 activity is enhanced by RNA helicase A. Cancer Res. 2006; 66:5574-81.

18. Bagnara GP, Serra M, Giovannini M, Badiali M, Stella M, Montaldi A, Tessarollo L, Cavazzana AO. Establishment and characterization of a primitive neuroectodermal tumor of bone continuous cell line (LAP-35). Int J Cell Cloning. 1990; 8:409-24.

19. Whang-Peng J, Triche TJ, Knutsen T, Miser J, KaoShan S, Tsai S, Israel MA. Cytogenetic characterization of selected small round cell tumors of childhood. Cancer Genet Cytogenet. 1986; 21:185-208.

20. Corrionero A, Minana B, Valcarcel J. Reduced fidelity of branch point recognition and alternative splicing induced by the anti-tumor drug spliceostatin A. Genes Dev. 2011; 25:445-59.

21. Nakajima T, Uchida C, Anderson SF, Lee CG, Hurwitz J, Parvin JD, Montminy M. RNA helicase A mediates association of CBP with RNA polymerase II. Cell. 1997; 90:1107-12.

22. Azzalin CM, Lingner J. The double life of UPF1 in RNA and DNA stability pathways. Cell Cycle. 2006; 5:1496-8. 
23. Valacca C, Bonomi S, Buratti E, Pedrotti S, Baralle FE, Sette C, Ghigna C, Biamonti G. Sam68 regulates EMT through alternative splicing-activated nonsense-mediated mRNA decay of the SF2/ASF proto-oncogene. J Cell Biol. 2010; 191:87-99.

24. Takeda Y, Caudell P, Grady G, Wang G, Suwa A, Sharp GC, Dynan WS, Hardin JA. Human RNA helicase $\mathrm{A}$ is a lupus autoantigen that is cleaved during apoptosis. J Immunol. 1999; 163:6269-74.

25. Rockx DA, Mason $\mathrm{R}$, van Hoffen A, Barton MC, Citterio E, Bregman DB, van Zeeland AA, Vrieling H, Mullenders LHF. UV-induced inhibition of transcription involves repression of transcription initiation and phosphorylation of RNA polymerase II. Proc Natl Acad Sci U S A. 2000; 97:10503-8.

26. Somesh BP, Reid J, Liu WF, Sogaard TM, ErdjumentBromage H, Tempst P, Svejstrup JQ. Multiple mechanisms confining RNA polymerase II ubiquitylation to polymerases undergoing transcriptional arrest. Cell. 2005; 121:913-23.

27. Laybourn PJ, Dahmus ME. Transcription-dependent structural changes in the C-terminal domain of mammalian RNA polymerase subunit IIa/o. J Biol Chem. 1989; 264:6693-8.

28. Shukla S, Oberdoerffer S. Co-transcriptional regulation of alternative pre-mRNA splicing. Biochim Biophys Acta. 2012; 1819:673-83.

29. Kornblihtt AR, Schor IE, Allo M, Dujardin G, Petrillo E, Munoz MJ. Alternative splicing: a pivotal step between eukaryotic transcription and translation. Nat Rev Mol Cell Biol. 2013; 14:153-65.

30. Batsche E, Yaniv M, Muchardt C. The human SWI/SNF subunit Brm is a regulator of alternative splicing. Nat Struct Mol Biol. 2006; 13:22-9.

31. Hsin JP, Manley JL. The RNA polymerase II CTD coordinates transcription and RNA processing. Genes Dev. 2012; 26:2119-37.

32. Marshall NF, Peng J, Xie Z, Price DH. Control of RNA polymerase II elongation potential by a novel carboxyl-terminal domain kinase. J Biol Chem. 1996; 271:27176-83.

33. Ip JY, Schmidt D, Pan Q, Ramani AK, Fraser AG, Odom DT, Blencowe BJ. Global impact of RNA polymerase II elongation inhibition on alternative splicing regulation. Genome Res. 2011; 21:390-401.

34. Fukuma M, Okita H, Hata J, Umezawa A. Upregulation of Id2, an oncogenic helix-loop-helix protein, is mediated by the chimeric EWS/ets protein in Ewing sarcoma. Oncogene. 2003; 22:1-9.

35. Erkizan HV, Kong Y, Merchant M, Schlottmann S, Barber-Rotenberg JS, Yuan L, Abaan OD, Chou TH, Dakshanamurthy S, Brown ML, Uren A, Toretsky JA.
A small molecule blocking oncogenic protein EWS-FLI1 interaction with RNA helicase A inhibits growth of Ewing's sarcoma. Nat Med. 2009; 15:750-6.

36. Patel SR. New agents in the treatment of soft-tissue sarcomas. Expert Opin Investig Drugs. 2000; 9:1545-51.

37. Starita LM, Horwitz AA, Keogh MC, Ishioka C, Parvin JD, Chiba N. BRCA1/BARD1 ubiquitinate phosphorylated RNA polymerase II. J Biol Chem. 2005; 280:24498-505.

38. Dujardin G, Lafaille C, de la Mata M, Marasco LE, Munoz MJ, Le Jossic-Corcos C, Corcos L, Kornblihtt AR. How slow RNA polymerase II elongation favors alternative exon skipping. Mol Cell. 2014; 54:683-90.

39. Linder P, Lasko PF, Ashburner M, Leroy P, Nielsen PJ, Nishi K, Schnier J, Slonimski PP. Birth of the D-E-A-D box. Nature. 1989; 337:121-2.

40. Rocak S, Linder P. DEAD-box proteins: the driving forces behind RNA metabolism. Nat Rev Mol Cell Biol. 2004; 5:232-41.

41. Lee CG, da Costa Soares V, Newberger C, Manova K, Lacy E, Hurwitz J. RNA helicase A is essential for normal gastrulation. Proc Natl Acad Sci U S A. 1998; 95:13709-13.

42. Jain A, Bacolla A, Chakraborty P, Grosse F, Vasquez KM. Human DHX9 helicase unwinds triple-helical DNA structures. Biochemistry. 2010; 49:6992-9.

43. Jain A, Bacolla A, Del Mundo IM, Zhao J, Wang G, Vasquez KM. DHX9 helicase is involved in preventing genomic instability induced by alternatively structured DNA in human cells. Nucleic Acids Res. 2013; 41:10345-10357.

44. Mills JR, Malina A, Lee T, Di Paola D, Larsson O, Miething C, Grosse F, Tang H, Zannis-Hadjopoulos M, Lowe SW, Pelletier J. RNAi screening uncovers Dhx9 as a modifier of ABT-737 resistance in an Emu-myc/Bcl-2 mouse model. Blood. 2013; 121:3402-12.

45. Friedemann J, Grosse F, Zhang S. Nuclear DNA helicase II (RNA helicase A) interacts with Werner syndrome helicase and stimulates its exonuclease activity. J Biol Chem. 2005; 280:31303-13.

46. Schlegel BP, Starita LM, Parvin JD. Overexpression of a protein fragment of RNA helicase A causes inhibition of endogenous BRCA1 function and defects in ploidy and cytokinesis in mammary epithelial cells. Oncogene. 2003; 22:983-91.

47. Christmann M, Tomicic MT, Origer J, Aasland D, Kaina B. c-Fos is required for excision repair of UV-light induced DNA lesions by triggering the re-synthesis of XPF. Nucleic Acids Res. 2006; 34:6530-9.

48. Grier HE, Krailo MD, Tarbell NJ, Link MP, Fryer CJ, Pritchard DJ, Gebhardt MC, Dickman PS, Perlman EJ, Meyers PA, Donaldson SS, Moore S, Rausen AR, Vietti TJ, Miser JS. Addition of ifosfamide and etoposide 
to standard chemotherapy for Ewing's sarcoma and primitive neuroectodermal tumor of bone. N Engl J Med. 2003; 348:694-701.

49. Hu-Lieskovan S, Heidel JD, Bartlett DW, Davis ME, Triche TJ. Sequence-specific knockdown of EWS-FLI1 by targeted, nonviral delivery of small interfering RNA inhibits tumor growth in a murine model of metastatic Ewing's sarcoma. Cancer Res. 2005; 65:8984-92.

50. Kovar H, Ban J, Pospisilova S. Potentials for RNAi in sarcoma research and therapy: Ewing's sarcoma as a model. Semin Cancer Biol. 2003; 13:275-81.
51. Dudoit S, Gentleman RC, Quackenbush J. Open source software for the analysis of microarray data. Biotechniques. 2003; 45-51.

52. Ritchie ME, Silver J, Oshlack A, Holmes M, Diyagama D, Holloway A, Smyth GK. A comparison of background correction methods for two-colour microarrays. Bioinformatics. 2007; 23:2700-7.

53. Yang YH1, Dudoit S, Luu P, Lin DM, Peng V, Ngai J, Speed TP. Normalization for cDNA microarray data: a robust composite method addressing single and multiple slide systematic variation. Nucleic Acids Res. 2002; 30:e15. 